TI 2012-052/1

Tinbergen Institute Discussion Paper
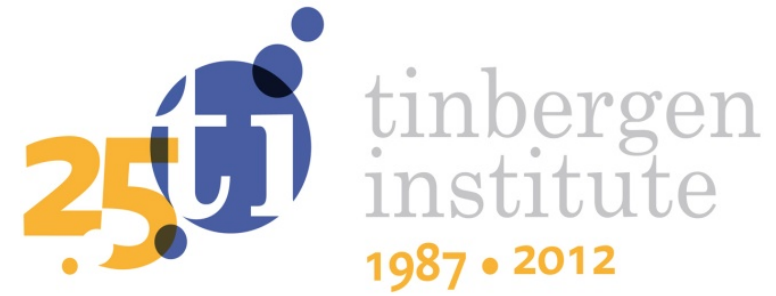

\title{
Share Functions for Cooperative Games with Levels Structure of Cooperation
}

\author{
Mikel Alvarez-Mozos' \\ René van den Brink² \\ Gerard van der Laan² \\ Oriol Tejada ${ }^{3}$
}

1 University of Santiago de Compostela;

2 Faculty of Economics and Business Administration, VU University of Amsterdam, and Tinbergen Institute;

3 ETH, Zürich. 
Tinbergen Institute is the graduate school and research institute in economics of Erasmus University Rotterdam, the University of Amsterdam and VU University Amsterdam.

More TI discussion papers can be downloaded at http://www.tinbergen.nl

Tinbergen Institute has two locations:

Tinbergen Institute Amsterdam

Gustav Mahlerplein 117

1082 MS Amsterdam

The Netherlands

Tel.: +31(0)205251600

Tinbergen Institute Rotterdam

Burg. Oudlaan 50

3062 PA Rotterdam

The Netherlands

Tel.: +31(0)10 4088900

Fax: $+31(0) 104089031$

Duisenberg school of finance is a collaboration of the Dutch financial sector and universities, with the ambition to support innovative research and offer top quality academic education in core areas of finance.

DSF research papers can be downloaded at: http://www.dsf.nl/

Duisenberg school of finance

Gustav Mahlerplein 117

1082 MS Amsterdam

The Netherlands

Tel.: +31(0)20 5258579 


\title{
Share functions for cooperative games with levels structure of cooperation
}

\author{
M. Álvarez-Mozos ${ }^{1}$, R. van den Brink², G. van der Laan ${ }^{3}$, and O. Tejada*4 \\ ${ }^{1}$ Department of Statistics and Operations Research, Faculty of Mathematics, University of Santiago de \\ Compostela, Spain. \\ ${ }^{2,3}$ Department of Econometrics and Tinbergen Institute, VU University Amsterdam, The Netherlands. \\ ${ }^{4}$ CER-ETH Center of Economic Research, ETH Zurich, Switzerland
}

May 9, 2012

\begin{abstract}
In a standard TU-game it is assumed that every subset of the player set $N$ can form a coalition and earn its worth. One of the first models where restrictions in cooperation are considered is the one of games with coalition structure of Aumann and Drèze (1974). They assumed that the player set is partitioned into unions and that players can only cooperate within their own union. Owen (1977) introduced a value for games with coalition structure under the assumption that also the unions can cooperate among them. Winter (1989) extended this value to games with levels structure of cooperation, which consists of a game and a finite sequence of partitions defined on the player set, each of them being coarser than the previous one.

A share function for TU-games is a type of solution that assigns to every game a vector whose components add up to one, and thus they can be interpreted as players' shares in the worth to be allocated. Extending the approach to games with coalition structure developed in van den Brink and van der Laan (2005), we introduce a class of share functions for games with levels structure of cooperation by defining, for each player and each level, a standard TU-game. The share given to each player is then defined as the product of her shares in the games at every level. We show several desirable properties and provide axiomatic characterizations of this class of LS-share functions.
\end{abstract}

\footnotetext{
*Corresponding author's e-mail address: toriol@ethz.ch.
} 
Keywords: cooperative game, Shapley value, coalition structure, share functions, levels structure of cooperation

\section{Introduction}

A cooperative game with transferable utility, or simply a TU-game, is a finite set of players $N$ and for any subset (coalition) of players a worth representing the total payoff that the coalition can obtain by cooperating. A value function for TU-games is a function that assigns to every TU-game with $n$ players an $n$-dimensional vector representing a distribution of payoffs among the players. A value function is efficient if for every game it distributes exactly the worth of the 'grand coalition', $N$, over all players. The most famous efficient value function is the Shapley value (Shapley, 1953). An example of a value function that is not efficient is the Banzhaf value (Banzhaf, 1965; Owen, 1975; Dubey and Shapley, 1979). Since the Banzhaf value is not efficient it is not adequate in allocating the worth $v(N)$. In order to allocate $v(N)$ according to the Banzhaf value, van den Brink and van der Laan (1998) characterize the normalized Banzhaf value, which distributes the worth $v(N)$ proportional to the Banzhaf values of the players.

A different approach to efficiently allocate the worth $v(N)$ is described in van der Laan and van den Brink (1998), who introduce share functions as an alternative type of solution for TU-games. A share vector for an $n$-player game is an $n$-dimensional real vector whose components add up to one. The $i^{\text {th }}$ component is player $i$ 's share in the total payoff that is to be distributed among the players. A share function assigns such a share vector to every game. The share function corresponding to the Shapley value is the Shapley share function, which is obtained by dividing the Shapley value of each player by $v(N)$, i.e., by the sum of the Shapley values of all players. Similarly, the Banzhaf share function is obtained by dividing the Banzhaf -or normalized Banzhaf- value by the corresponding sum of payoffs over all players. One advantage of share functions over value functions is that share functions avoid the "efficiency issue", i.e., they avoid the question of what is the final worth to be distributed over the players. This yields some major simplifications. For example, although the Banzhaf and normalized Banzhaf value are very different value functions (e.g. the Banzhaf value satisfies linearity and the dummy player property which are not satisfied by the normalized Banzhaf value), they correspond to the same Banzhaf share function. Another main advantage of share functions has been discovered by Pekec (2001), who shows that on a ratio scale meaningful statements can be made for a certain class of share functions, whereas all statements with respect to value 
functions are meaningless. ${ }^{1}$

In a standard TU-game it is assumed that every subset of the player set $N$ is a feasible coalition and can earn its worth. However, there are many real life situations in which cooperation is restricted, e.g. there is a priori information about the behavior of the players or only partial cooperation is possible due to environmental restrictions. The games with coalition structure proposed in Aumann and Drèze (1974) are among the first models in which restrictions in cooperation are considered. They assume that the player set is partitioned and that players can only cooperate with other players that belong to the same element of the partition. Such a partition is called a coalition structure and the elements of the coalition structure are called unions. Owen (1977) allows that a subset of players within a union can also cooperate with players of other unions, but only if all players of the other unions agree. His proposed value, known as the Owen value, can be introduced in several ways. We can define an external game or quotient game as a game where the unions form the player set, and the worth of a coalition of unions simply equals the worth of that 'union of unions' in the original game. Then, for every union, an internal game is defined where the worth of every subset of players within that union equals the Shapley value of that subset in the modified quotient game obtained by replacing that union by the specific subset. Taking the Shapley value of this internal game yields the Owen value of the game with coalition structure. This value satisfies the (consistency) property that the sum of the payoffs assigned to the players in a particular union equals the Shapley value of that union in the quotient game.

A straightforward example shows that the existence of a coalition structure might have a significant impact on the outcome. Suppose that, in a simple majority voting game, the coalition structure consists of two unions of players. If the two unions have not the same number of votes, then, by definition of the voting game, one of the unions has the majority. Ignoring the coalition structure, we might apply the Shapley value and typically this results in an outcome in which every player is assigned a positive payoff (power). Taking into account the coalition structure, the Owen value assigns the full worth of the grand coalition, $v(N)=1$, to the players of the majority coalition and, thus, all players in the minority coalition get zero payoff.

Similar insights might be obtained in situations with more complex restrictions to the cooperation among players. In Courtin (2011) the distribution of power in the European Parliament is analyzed when the a priori information that limits the cooperation among parties can

\footnotetext{
${ }^{1} \mathrm{~A}$ statement for a TU-game is meaningful on a ratio scale if the statement is also true if we multiply the relevant variables by the same constant, see Pekec (2001).
} 
be represented by two arbitrary partitions of the set of parties, that respond respectively to an economic and a political criterion. Whereas the Aumann-Drèze coalition structure is a partition of the grand coalition, in the model of Courtin (2011) the resulting coalition structure is a collection of unions with possibly non-empty intersection. These games, with the so-called coalition configuration structure, are introduced in Albizuri et al. (2006) and Albizuri and Aurrekoetxea (2006).

In this paper we consider a different structure that restricts the cooperation possibilities among the agents. To illustrate the validity of the model, we briefly discuss its possible application to the European Parliament. This chamber is, together with the "Council of Ministers" and the "European Commission", one of the main European political institutions. It consists of 754 delegates from 27 different countries. Members of each country are organized within national parties, and parties from different countries are organized in seven parliamentary groups. The three largest groups are the European People's Party, the Socialists and Democrats, and the Alliance of Liberals and Democrats. Some of these groups are further divided into (formal) smaller subgroups, e.g. the latter Alliance consists of two subgroups: the Liberals and the Democrats. Moreover, it might also happen that on particular issues other (informal) subgroups are formed, e.g. according to geographical proximity among national parties. Considering the structure from top to down, we observe that first there is a coalition structure consisting of the seven parliamentary groups. Then, within such a group there is a second coalition structure consisting of the subgroups. Next, within each subgroup there is again a coalition structure consisting of national parties. To study the power of an individual member of the parliament it seems necessary to take into account this organizational structure.

Games with such a structure have been considered in Winter (1989), who extends games with coalition structure to the so-called games with levels structure of cooperation. This model consists of a game and a finite sequence of partitions defined on the player set, each of them being coarser than the previous one. Considering the 'highest' level as a coalition structure, each union is again partitioned so that players within a union in this next level are somehow closer to each other than to other players in the union, who are still closer than players outside the union in the highest level. Again, this partition of the unions can be partitioned further, and so on and so forth. For this model Winter (1989) suggests a solution that generalizes the Owen value for games with coalition structure, and thus also generalizes the Shapley value for standard TU-games. Recently, Álvarez-Mozos and Tejada (2011) defined an extension of the (non efficient) Banzhaf value for games with levels structure of cooperation, and provided 
comparable axiomatizations of this value and Winter's Shapley type value.

In van den Brink and van der Laan (2005) it is shown that share functions have additional desirable properties when applying them to games with coalition structure. For every element in the class of share functions mentioned above, they defined a share function for games with coalition structure, taking for every player the product of her share in the internal game and the share of her union in the external (quotient) game. These share functions satisfy the consistency property that the sum of the shares over players in one union equals the share of the union in the quotient game. Applying the Shapley share function twice yields the Owen value (after multiplying by $v(N)$ ). However, applying the Banzhaf share function twice ${ }^{2}$ yields a different solution than the Banzhaf value for games with coalition structure considered in Owen (1981). In van den Brink and van der Laan (2005), see also Andjiga and Courtin (2010), two axiomatizations of such a class of share functions are given, one using the multiplication property, and another one using the consistency property relating the payoffs of players within a union to the payoff of the union in the quotient game.

The results of van den Brink and van der Laan (2005) show that taking the product of two shares is a natural method to define solutions for games with coalition structure. In this paper we extend this argument to games with levels structure of cooperation. We introduce a class of LS-share functions by defining a standard TU-game for every union at every level, and then assigning to every player the product of her shares over the corresponding games at every level.

We show several desirable properties of these solutions. Two fundamental properties are (i) they yield the corresponding solution for standard TU-games in case the levels structure is trivial (meaning that the grand coalition $N$ is partitioned immediately in a coalition structure consisting of all singletons of $N$ ), and (ii) the sum of the shares of the players in a union equals the share of the union in the game played among the unions of this level (consistency). We provide axiomatizations of these share functions, extending those that are known for games with coalition structure. Moreover, we use weaker versions of some of the axioms used for games with coalition structure by requiring them only for trivial structures. Besides generalizing those two axiomatizations, we introduce a new type of axiom which we refer to as $\mu$-fairness.

The class of LS-share functions introduced in this paper is parametrized by real valued $\mu$-functions on the set of TU-games. These functions are introduced in van der Laan and van den Brink (1998). The first result of this paper characterizes the class of $\mu$-functions that are

\footnotetext{
${ }^{2}$ Recall that the Banzhaf value and normalized Banzhaf value yield the same Banzhaf share function.
} 
positive, symmetric, and additive.

The paper is organized as follows. Section 2 contains preliminaries on TU-games. In Section 3 we review share functions and present the result which characterizes all positive, additive, and symmetric $\mu$-functions, and the corresponding share functions for TU-games. In Section 4 we introduce the class of LS-share functions for games with levels structure of cooperation, we derive the first properties, and we give special attention to the Shapley levels share function. Finally, in Section 5 we provide the axiomatic characterizations.

\section{Preliminaries}

A cooperative game with transferable utility is a pair $(N, v)$, where $N$ is a finite set of players and $v$, the characteristic function, is a real valued function on $2^{N}=\{S: S \subseteq N\}$ with $v(\emptyset)=0$. Along the paper we use the $|\cdot|$ operator to denote the cardinality of a finite set, i.e., $|S|$ is the number of players in $S$, for any $S \subseteq N$. Alternatively, sometimes we use lowercase letters to denote cardinalities, and thus $s=|S|$ for any $S \subseteq N$. A game $(N, v)$ is called monotone if for every $S, T \subseteq N$ with $S \subseteq T$, it holds that $v(S) \leq v(T)$. That is, monotone games are those in which the cooperation among players is never pernicious. Notice that the class of monotone games is a quite wide class of games. We denote by $\mathcal{G}$ the set of all monotone games. Since the whole paper deals with monotone games, henceforth we will simply say game instead of monotone game. For an arbitrary finite set $K$, we denote by $\mathbb{R}^{K}$ to the $|K|$-dimensional Euclidean space with elements $x \in \mathbb{R}^{K}$ having components $x_{i}, i \in K$.

For each $S \subseteq N$ and $i \in N$, we will write $S \cup i$ instead of $S \cup\{i\}$ and $S \backslash i$ instead of $S \backslash\{i\}$. For a pair of games $(N, v),(N, w) \in \mathcal{G}$, the game $(N, z)$ with $z=v+w$ is defined by $z(S)=v(S)+w(S)$ for all $S \subseteq N$. We denote by $\left(N, v_{0}\right)$ the null game with player set $N$, i.e., $v_{0}(S)=0$ for all $S \subseteq N$. For every nonempty $T \subseteq N$ and $(N, v) \in \mathcal{G}$, we denote by $\left(T, v_{\mid T}\right)$ the subgame on $T$ given by $v_{\mid T}(S)=v(S)$ for all $S \subseteq T$. Further, given $S \in 2^{N}$, the unanimity game

with carrier $S,\left(N, u^{S}\right)$, is defined by $u^{S}(T)=1$ if $S \subseteq T$, and $u^{S}(T)=0$ otherwise. Notice that $\left(N, u^{S}\right) \in \mathcal{G}$ for every $S \in 2^{N}$.

Given $(N, v) \in \mathcal{G}$, a player $i \in N$ is a dummy if $v(S \cup i)=v(S)+v(i)$ for all $S \subseteq N \backslash i$, that is, if all her marginal contributions are equal to $v(i)$. A player $i \in N$ is called a null player if she is a dummy and $v(i)=0$. Two players $i, j \in N$ are symmetric if $v(S \cup i)=v(S \cup j)$ for all $S \subseteq N \backslash\{i, j\}$, that is, if their marginal contributions to each coalition coincide.

A value function, or shortly value, on $\mathcal{G}$ is a map $f$ that assigns to every game $(N, v) \in \mathcal{G}$ 
a vector $f(N, v) \in \mathbb{R}^{N}$. The following definitions provide the explicit expressions of two wellknown values in the literature.

\section{Definition 2.1. (Shapley, 1953)}

The Shapley value, Sh, assigns to any game $(N, v)$, a vector in $\mathbb{R}^{N}$ defined as

$$
\operatorname{Sh}_{i}(N, v)=\sum_{S \subseteq N \backslash i} \frac{s !(n-s-1) !}{n !}[v(S \cup i)-v(S)], \quad i \in N .
$$

\section{Definition 2.2. (Banzhaf, 1965; Owen, 1975)}

The Banzhaf value, Ba, assigns to any game $(N, v)$ a vector in $\mathbb{R}^{N}$ defined as

$$
\operatorname{Ba}_{i}(N, v)=\sum_{S \subseteq N \backslash i} \frac{1}{2^{n-1}}[v(S \cup i)-v(S)], \quad i \in N .
$$

By a share function we mean a map $\rho$ that assigns to every game $(N, v) \in \mathcal{G}$ a vector $\rho(N, v) \in \mathbb{R}^{N}$ such that $\sum_{i \in N} \rho_{i}(N, v)=1$. The component $\rho_{i}(N, v)$ represents player $i$ 's share in the worth to be distributed. Consequently, a share function $\rho$ may be obtained from each value $f$ on $\mathcal{G}$ by defining for every $(N, v) \in \mathcal{G}$ and every player $i \in N$ its share $\rho_{i}(N, v)=\frac{f_{i}(N, v)}{\sum_{j \in N} f_{j}(N, v)}$. In the next definition the share functions obtained from Sh and Ba are presented.

\section{Definition 2.3 .}

(i) Given a game $(N, v) \in \mathcal{G}$, the Shapley share function, $\rho^{\text {Sh }}$, assigns to any game $(N, v)$ a vector in $\mathbb{R}^{N}$ defined as $\rho_{i}^{\mathrm{Sh}}(N, v)=\frac{\mathrm{Sh}_{i}(N, v)}{v(N)}, i \in N$, if $v \neq v_{0}$, and $\rho_{i}^{\mathrm{Sh}}\left(N, v_{0}\right)=\frac{1}{|N|}, i \in N$.

(ii) Given a game $(N, v) \in \mathcal{G}$, the Banzhaf share function, $\rho^{\mathrm{Ba}}$, assigns to any game $(N, v)$ a vector in $\mathbb{R}^{N}$ defined as $\rho_{i}^{\mathrm{Ba}}(N, v)=\frac{\mathrm{Ba}_{i}(N, v)}{\sum_{j \in N} \mathrm{Ba}_{j}(N, v)}, i \in N$, if $v \neq v_{0}$, and $\rho_{i}^{\mathrm{Ba}}\left(N, v_{0}\right)=\frac{1}{|N|}$, $i \in N$.

\section{Share functions}

In van der Laan and van den Brink (1998) a class of share functions for TU-games is defined and characterized based on real valued functions $\mu: \mathcal{G} \longrightarrow \mathbb{R}$. The Shapley and Banzhaf share functions are included in this class for appropriate choices of $\mu$-functions. In this section we reconsider and extend the results of van der Laan and van den Brink (1998). In Subsection 3.1 we propose a set of properties for $\mu$-functions that will allow us to write them as a sum of weighted marginal contributions where the weights are of a certain type. In Subsection 3.2 we merge the aforesaid result with the characterization of van der Laan and van den Brink (1998). 


\subsection{Real valued functions on $\mathcal{G}$}

We consider some properties that real valued functions on $\mathcal{G}, \mu: \mathcal{G} \rightarrow \mathbb{R}$, might satisfy. By $\mathcal{G}_{0}$ we denote the class of null games $\left(N, v_{0}\right)$, and by $\mathcal{G}_{+}=\mathcal{G} \backslash \mathcal{G}_{0}$ the class of non-null games. Let $\Pi(N)$ denote the set of all permutations of $N$ and, for any $\pi \in \Pi(N)$ and $(N, v) \in \mathcal{G}$, define the game $(N, \pi v)$ where $(\pi v)(T)=v(\pi(T))$ for all $T \subseteq N$. We say that

(a) $\mu$ is positive if $\mu\left(N, v_{0}\right)=0$ and $\mu(N, v)>0$ for every $(N, v) \in \mathcal{G}_{+}$.

(b) $\mu$ is additive if for every pair of games $(N, v),(N, w) \in \mathcal{G}$, it holds that $\mu(N, v+w)=$ $\mu(N, v)+\mu(N, w)$.

(c) $\mu$ is symmetric if for every pair of symmetric players $i, j \in N$ in $(N, v) \in \mathcal{G}$ and every $S \subseteq N \backslash\{i, j\}$, it holds that $\mu\left(S \cup i, v_{\mid S \cup i}\right)=\mu\left(S \cup j, v_{\mid S \cup j}\right)$.

(d) $\mu$ is anonymous if, for every game $(N, v) \in \mathcal{G}$, every permutation $\pi \in \Pi(N)$, and every nonempty subset $S \subseteq N$, it holds that $\mu\left(S, v_{\mid S}\right)=\mu\left(\pi(S),\left(\pi^{-1} v\right)_{\mid \pi(S)}\right)$.

In van der Laan and van den Brink (1998) the first two properties are used to define a family of $\mu$-functions on a fixed player set. It is there proved that a certain characterization result for share functions, which makes use of a property called $\mu$-additivity, holds as long as $\mu$ is positive, additive, and anonymous ${ }^{3}$. In van den Brink and van der Laan (1999) their result is extended to a variable player set using symmetry instead of anonymity. Nevertheless, the formulation of anonymity seems to be a more natural way of requiring that the identity of players does not play a role. In the Appendix (see Proposition 6.1) it is shown that symmetry and anonymity are in fact equivalent properties. This implies that, although we use the anonymity formulation henceforth, we are actually considering exactly the same class of functions as in van der Laan and van den Brink (1998) for a fixed player set.

We next show that, except for the equivalence between symmetry and anonymity, there is no logical relation among the above properties.

Proposition 3.1. Additivity, positivity, and anonymity are independent properties.

Proof. First, $\mu(N, v)=v(N)^{2}$ satisfies positivity and anonymity but not additivity. Second, $\mu(N, v)=-v(N)$ satisfies additivity and anonymity but not positivity. Third, take a

\footnotetext{
${ }^{3}$ The necessity for anonymity is mentioned in Theorem 25 and Footnote 6 in van den Brink and van der Laan (1999).
} 
particular player $j$ and define $\mu$ as

$$
\mu(N, v)= \begin{cases}2 v(N) & \text { if } j \in N \\ v(N) & \text { otherwise }\end{cases}
$$

Then $\mu$ satisfies additivity and positivity but not anonymity.

The following result shows that any real valued function $\mu$ that is positive, additive, and anonymous can be written as a linear combination of marginal contributions with the weights depending only on the sizes of the corresponding coalitions.

Theorem 3.2. Let $\mu$ be a real valued function on $\mathcal{G}$. Then, the two following statements are equivalent:

(a) $\mu$ is positive, additive, and anonymous.

(b) There is a unique family of strictly positive weights, $\left\{\omega_{\mu}^{n, s} \in \mathbb{R}: n \in \mathbb{N}\right.$ and $s \in\{0,1, \ldots, n-$ $1\}$ \} such that, for every $(N, v) \in \mathcal{G}$,

$$
\mu(N, v)=\sum_{i \in N} \sum_{S \subseteq N \backslash i} \omega_{\mu}^{|N|,|S|}[v(S \cup i)-v(S)] .
$$

Proof. We first prove the implication $(b) \Longrightarrow(a)$. First, since the weights are strictly positive and the games in $\mathcal{G}$ are monotone, $\mu$ is positive. Second, since $\mu(N, v)$, as defined in eq. (1), is linear on each $v(S), S \subseteq N, \mu$ is additive. Third, since the weights depend only on the cardinality of the sets, $\mu(N, v)$ is anonymous.

Next we prove the implication $(a) \Longrightarrow(b)$. Let $N$ and $\mu: \mathcal{G} \longrightarrow \mathbb{R}$ be fixed. We denote by $\mathcal{G}^{N}$ the set of games with the fixed player set $N$. It is well known that $\mathcal{G}^{N}$ is a cone in the euclidean space of dimension $2^{n}-1$. As a consequence, $\mu$ can be cast as the restriction of a map $\mu^{*}: \mathbb{R}^{2^{n}-1} \longrightarrow \mathbb{R}$. Moreover, observe that $\mathcal{G}^{N}$ has positive measure in $\mathbb{R}^{2^{n}-1}$ because $\left\{\left(N, u^{S}\right)\right\}_{\emptyset \neq S \subseteq N}$ is a basis of $\mathbb{R}^{2^{n}-1}$ and for every $S \subseteq N,\left(N, u^{S}\right) \in \mathcal{G}$. Therefore, since $\mu$ is positive, $\mu^{*}$ is bounded from below in a set of positive measure. From the solution of the Cauchy equation for several variables applied to $\mu$ (see Proposition 1 at page 35 in Aczél and Dhombres (1989) for instance), for every $\emptyset \neq S \subseteq N$, there are scalars $a^{S} \in \mathbb{R}$, such that, for all $(N, v) \in \mathcal{G}^{N}$,

$$
\mu(N, v)=\mu^{*}(N, v)=\sum_{\emptyset \neq S \subseteq N} a^{S} v(S) .
$$


Note that the argument above applies for any given $\mu$ and $N$. Hence, for every positive, additive, and anonymous $\mu$, every finite set of players $N$, and every coalition $\emptyset \neq S \subseteq N$ there are scalars $a_{\mu}^{N, S}$ such that, for every $(N, v) \in \mathcal{G}$,

$$
\mu(N, v)=\sum_{\emptyset \neq S \subseteq N} a_{\mu}^{N, S} v(S) .
$$

Next, let $N$ and $N^{\prime}$ be two sets of players, $\emptyset \neq S \subseteq N$, and $S^{\prime} \subseteq N^{\prime}$ such that $|S|=\left|S^{\prime}\right|$. Let $\pi \in \Pi\left(N \cup N^{\prime}\right)$ be a permutation that exchanges $S$ with $S^{\prime}$ and $N$ with $N^{\prime}$. By anonymity,

$$
\mu\left(N, u_{\mid N}^{S}\right)=\mu\left(\pi(N),\left(\pi^{-1} u^{S}\right)_{\mid \Pi(N)}\right)=\mu\left(N^{\prime}, u_{\mid N^{\prime}}^{S^{\prime}}\right) .
$$

We prove that $a_{\mu}^{N, S}=a_{\mu}^{N^{\prime}, S^{\prime}}$ by reverse induction on the cardinality of $S$.

Let $S=N$ and $S^{\prime}=N^{\prime}$. Then, we have that $a_{\mu}^{N^{\prime}, N^{\prime}}=\mu\left(N^{\prime}, u_{\left.\right|_{N^{\prime}}}^{N^{\prime}}\right)=\mu\left(N, u_{\left.\right|_{N}}^{N}\right)=a_{\mu}^{N, N}$, where the first and last equality follow from eq. (2) and the second equality from eq. (3). Next, for some $s<n, T \subseteq N$ and $T^{\prime} \subseteq N^{\prime}$, assume that $a_{\mu}^{N, T}=a_{\mu}^{N^{\prime}, T^{\prime}}$ whenever $|T|=\left|T^{\prime}\right|>s$. Take $S \subsetneq N$ and $S^{\prime} \subsetneq N^{\prime}$ with $|S|=\left|S^{\prime}\right|=s>0$. By eq. (2) and eq. (3),

$a_{\mu}^{N^{\prime}, S^{\prime}}+\sum_{\left\{T^{\prime} \subseteq N^{\prime} \mid S^{\prime} \subsetneq T^{\prime}\right\}} a_{\mu}^{N^{\prime}, T^{\prime}}=\sum_{\left\{T^{\prime} \subseteq N^{\prime} \mid S^{\prime} \subseteq T^{\prime}\right\}} a_{\mu}^{N^{\prime}, T^{\prime}}=\sum_{\{T \subseteq N \mid S \subseteq T\}} a_{\mu}^{N, T}=a_{\mu}^{N, S}+\sum_{\{T \subseteq N \mid S \subsetneq T\}} a_{\mu}^{N, T}$.

By the induction hypothesis we have that for every $T^{\prime} \subseteq N^{\prime}$ with $S^{\prime} \subsetneq T^{\prime}$ and every $T \subseteq N$ with $S \subsetneq T$ such that $|S|=\left|S^{\prime}\right|, a_{\mu}^{N^{\prime}, T^{\prime}}=a_{\mu}^{N, T}$. Thus, eq. (4) leads to $a_{\mu}^{N, S}=a_{\mu}^{N^{\prime}, S^{\prime}}$. Thus, there exist numbers $a_{\mu}^{n, s}$, with $n \in \mathbb{N}$ and $s \in\{1, \ldots, n\}$, such that, for every $(N, v) \in \mathcal{G}$,

$$
\mu(N, v)=\sum_{\emptyset \neq S \subseteq N} a_{\mu}^{n, s} v(S) .
$$

Analogously to Corollary 3.8 of van der Laan and van den Brink (1998), for every $n \in \mathbb{N}$ and $s \in\{0, \ldots, n-1\}$, we recursively define the parameters $w_{\mu}^{n, s}$, by $\omega_{\mu}^{n, n-1}=\frac{1}{n} a_{\mu}^{n, n}$, and $\omega^{n, s}=\frac{1}{s+1}\left(a_{\mu}^{n, s+1}+(n-s-1) \omega_{\mu}^{n, s+1}\right)$ for all $s \in\{0, \ldots, n-2\}$. Then, we can rewrite eq. (5) as

$$
\mu(N, v)=\sum_{i \in N} \sum_{S \subseteq N \backslash i} \omega_{\mu}^{n, s}[v(S \cup i)-v(S)] .
$$

Hence, we have proved the existence of a family of weights, $\left\{\omega_{\mu}^{n, s} \in \mathbb{R}: n \in \mathbb{N}\right.$ and $s \in\{0, \ldots, n-$ $1\}$ such that eq. (6) holds for every $(N, v) \in \mathcal{G}$.

To prove the uniqueness of the weights, let $\left\{\omega_{\mu}^{n, s}\right\}_{n \in \mathbb{N}, s \in\{0, \ldots, n-1\}}$ and $\left\{\delta_{\mu}^{n, s}\right\}_{n \in \mathbb{N}, s \in\{0, \ldots, n-1\}}$ be two systems of weights that satisfy eq. (6). For each $n \in \mathbb{N}$ and $s \in\{0, \ldots, n-1\}$, let $\left(N, v^{s}\right) \in \mathcal{G}$ with $|N|=n$ be defined by $v^{s}(T)=1$ if $|T|>s$ and $v^{s}(T)=0$ otherwise. Then, $0=\mu\left(N, v^{s}\right)-\mu\left(N, v^{s}\right)=\sum_{i \in N} \sum_{S \subseteq N \backslash i}\left(\omega_{\mu}^{n,|S|}-\delta_{\mu}^{n,|S|}\right)\left[v^{s}(S \cup i)-v^{s}(S)\right]=\left(\omega_{\mu}^{n, s}-\delta_{\mu}^{n, s}\right) \cdot n \cdot\left(\begin{array}{c}n-1 \\ s\end{array}\right)$, 
which implies that $\omega_{\mu}^{n, s}=\delta_{\mu}^{n, s}$ for any $n \in \mathbb{N}$ and $s \in\{0, \ldots, n-1\}$. Hence, the system of weights is unique.

Finally, to prove that the weights are positive, notice that the game $\left(N, v^{s}\right) \in \mathcal{G}$ is not a null game. Therefore,

$$
0<\mu\left(N, v^{s}\right)=\sum_{i \in N} \sum_{S \subseteq N \backslash i} \omega_{\mu}^{n,|S|}\left[v^{s}(S \cup i)-v^{s}(S)\right]=\omega_{\mu}^{n, s} \cdot n \cdot\left(\begin{array}{c}
n-1 \\
s
\end{array}\right),
$$

which implies that $\omega_{\mu}^{n, s}>0$ for any $n \in \mathbb{N}$ and $s \in\{0, \ldots, n-1\}$.

Two important examples of additive, positive, and anonymous real valued functions are $\mu^{\mathrm{Sh}}(N, v)=v(N)$ and $\mu^{\mathrm{Ba}}(N, v)=\sum_{S \subseteq N}(2 s-n) v(S)$. They induce respectively the two families of weights defined for every $n \in \mathbb{N}$ and $s \in\{0, \ldots, n-1\}$ by

$$
\omega_{\mathrm{Sh}}^{n, s}=\omega_{\mu^{\mathrm{Sh}}}^{n, s}=\frac{s !(n-s-1) !}{n !} \quad \text { and } \quad \omega_{\mathrm{Ba}}^{n, s}=\omega_{\mu^{\mathrm{Ba}}}^{n, s}=\frac{1}{2^{n-1}} .
$$

\subsection{A characterization of share functions}

In this subsection we focus on share functions. We start by considering three properties that a share function might satisfy.

NPP A share function $\rho$ satisfies the Null Player Property if, for every $(N, v) \in \mathcal{G}_{+}$and every null player $i \in N$ in $(N, v)$, it holds that $\rho_{i}(N, v)=0$.

SYM A share function $\rho$ satisfies Symmetry if, for every $(N, v) \in \mathcal{G}$ and every pair $i, j \in N$ of symmetric players in $(N, v)$, it holds that $\rho_{i}(N, v)=\rho_{j}(N, v)$.

$\mu$-ADD Let $\mu: \mathcal{G} \rightarrow \mathbb{R}$. A share function $\rho$ satisfies $\mu$-Additivity if, for every pair of games $(N, v),(N, w) \in \mathcal{G}$, it holds that $\mu(N, v+w) \rho(N, v+w)=\mu(N, v) \rho(N, v)+\mu(N, w) \rho(N, w)$.

The two first properties, NPP and SYM, are the same as for value functions (except that here the null player property is not required for null games), whereas $\mu$-ADD generalizes the usual additivity property for value functions. The next result shows that the above three properties determine a unique share function and that none of the properties can be left out.

Proposition 3.3. Let $\mu: \mathcal{G} \rightarrow \mathbb{R}$ be positive, additive, and anonymous. Then, there is a unique share function $\rho^{\mu}$ satisfying NPP, SYM, and $\mu$-ADD. This share function is given by

$$
\rho_{i}^{\mu}(N, v)=\frac{\sum_{S \subseteq N \backslash i} \omega_{\mu}^{n, s}[v(S \cup i)-v(S)]}{\mu(N, v)}, \quad i \in N, \text { if } v \neq v_{0},
$$

and $\rho_{i}^{\mu}\left(N, v_{0}\right)=\frac{1}{n}, i \in N$. Moreover, the three properties are independent. 
Proof. We only prove the independence of the properties since the characterization result holds as a direct consequence of Theorem 25 in van den Brink and van der Laan (1999), Theorem 3.2 in Subsection 3.1 and Proposition 6.1 in the Appendix.

First, let $a, b$ be two different and fixed players. Since $\mu$ is additive, positive, and anonymous, we can write

$$
\mu(\{a, b\}, v)=\lambda_{1} \cdot v(a)+\lambda_{1} \cdot v(b)+\lambda_{2} \cdot v(a, b),
$$

for some $0<\lambda_{1}, \lambda_{2}$. Then we define $\rho$ as follows:

- If $N=\{a, b\}$ and $(N, v) \in \mathcal{G}_{+}$, then

$$
\left\{\begin{array}{l}
\rho_{a}(N, v)=\frac{1}{\mu(N, v)}\left[\lambda_{1} \cdot v(a)+\lambda_{2} \cdot(v(a, b)-v(b))\right], \\
\rho_{b}(N, v)=\frac{1}{\mu(N, v)}\left[\left(\lambda_{1}+\lambda_{2}\right) \cdot v(b)\right] .
\end{array}\right.
$$

- If $N \neq\{a, b\}$, then $\rho(N, v)=\rho^{\mu}(N, v)$.

It is straightforward to check that $\rho$ is a share function that satisfies NPP and $\mu$-ADD. However it does not satisfy SYM. To prove this last assertion, consider the game $(N, v) \in \mathcal{G}$ where $N=\{a, b\}$ and $v(a)=v(b)=v(a, b)=1$. It is clear that $a$ and $b$ are symmetric players in $(N, v)$ but

$$
\mu(N, v) \rho_{a}(N, v)=\lambda_{1} \neq \lambda_{1}+\lambda_{2}=\mu(N, v) \rho_{b}(N, v) .
$$

Second, let $\mu: \mathcal{G} \rightarrow \mathbb{R}$ and $\mu^{\prime}: \mathcal{G} \rightarrow \mathbb{R}, \mu \neq \mu^{\prime}$ be two positive, additive, and anonymous functions. Then, from the uniqueness of the characterization result, $\rho^{\mu^{\prime}}$ satisfies NPP and SYM but not $\mu$-ADD.

Third, let $\rho$ be defined for every $(N, v) \in \mathcal{G}$ and every $i \in N$ by $\rho_{i}(N, v)=1 / n$. Then, for any additive $\mu, \rho$ satisfies $\mu$-ADD and SYM but not NPP.

Note that the expression of $\rho^{\mu}$ given in Proposition 3.3 implies that all shares are nonnegative for any monotone game. It should also be noticed that Theorem 3.5 in van der Laan and van den Brink (1998) proves an even stronger result for a fixed player set. ${ }^{4}$ Given a $\mu$-function that is positive and anonymous, it is there shown that the characterization result for share functions holds if and only if $\mu$ is additive. This fact supports the need to consider $\mu$-functions that not only are positive and anonymous - properties that are very standard - but also additive. Note also that, when $\mu=\mu^{\mathrm{Sh}}$, the unique share function satisfying the corresponding properties is $\rho^{\mathrm{Sh}}$, whereas when $\mu=\mu^{\mathrm{Ba}}$, it is $\rho^{\mathrm{Ba}}$. Lastly, observe that $\mu^{\mathrm{Sh}}$-ADD is the additivity property used by Shapley (1953).

\footnotetext{
${ }^{4}$ As mentioned in van den Brink and van der Laan (1999), these results hold under symmetry of $\mu$.
} 


\section{Games with levels structure of cooperation}

In the model analyzed so far cooperation among agents was not limited. In the rest of the paper a restriction to the cooperation is introduced as a finite sequence of partitions defined on the player set, each of them being coarser than the previous one. The worths of each coalition together with the sequence of partitions are now the available data.

Let $N$ be a finite set of players. For some integer $k \geq 0$, Winter (1989) introduced a $k$-level structure of cooperation over $N$ being a sequence of partitions of $N, \underline{B}=\left\{B_{0}, \ldots, B_{k+1}\right\}$ such that $B_{0}=\{\{i\}: i \in N\}, B_{k+1}=\{N\}$, and, for each $r \in\{0, \ldots, k\}, B_{r+1}$ is coarser than $B_{r}$. That is to say, for each $r \in\{1, \ldots, k+1\}$ and each $S \in B_{r}$, there is $B \subseteq B_{r-1}$ such that $S=\cup_{U \in B} U$. Each $S \in B_{r}$ is called a union and $B_{r}$ is called the $r$-th level of $\underline{B}$. The levels $B_{0}$ and $B_{k+1}$ are added for notational convenience. We denote by $\underline{B_{0}}$ the trivial levels structure with $k=0$, i.e., $\underline{B_{0}}=\left\{B_{0}, B_{1}\right\}$ with $B_{0}=\{\{i\}\}_{i \in N}$ and $B_{1}=\{N\}$. We further denote by $\mathcal{L}(N)$ the set of all levels structures of cooperation over the set $N$.

Example 4.1. Take $N=\{1,2,3,4,5\}$. Then $\underline{B} \in \mathcal{L}(N)$ given by $B_{0}=\{\{1\},\{2\},\{3\},\{4\},\{5\}\}$, $B_{1}=\{\{1,2\},\{3\},\{4,5\}\}, B_{2}=\{\{1,2\},\{3,4,5\}\}$ and $B_{3}=\{\{1,2,3,4,5\}\}$ is a 2-level structure of cooperation over $N$.

A game with levels structure of cooperation is a triple $(N, v, \underline{B})$, where $(N, v) \in \mathcal{G}$ and $\underline{B} \in \mathcal{L}(N)$. We denote by $\mathcal{G} \mathcal{L}$ the set of all games with levels structure of cooperation. Notice that a game with the trivial levels structure $\underline{B_{0}}$ corresponds to a standard transferable utility game and that a game with 1-level structure of cooperation $\underline{B}=\left\{B_{0}, B_{1}, B_{2}\right\}$ corresponds to a game with coalition structure as introduced in Aumann and Drèze (1974), also known as game with a priori unions in Owen (1977).

On the one hand, given $(N, v, \underline{B}) \in \mathcal{G L}$ with $\underline{B}=\left\{B_{0}, \ldots, B_{k+1}\right\}$, for each $r \in\{0, \ldots, k\}$ we define the $r^{t h}$ - union level game $\left(B_{r}, v^{r}, \underline{B^{r}}\right) \in \mathcal{G L}$ induced from $(N, v, \underline{B})$ as the game with $k-r$ levels structure of cooperation with the elements of $B_{r}$ as players, characteristic function $v^{r}$ given by $v^{r}(S)=v\left(\bigcup_{U \in S} U\right)$ for any coalition $S \subseteq B_{r}$, and with levels structure $\underline{B^{r}}=\left\{B_{0}^{r}, \ldots, B_{k-r+1}^{r}\right\}$ given by $B_{0}^{r}=\left\{\{U\}: U \in B_{r}\right\}, B_{s}^{r}=\left\{\left\{U \in B_{r}: U \subseteq U^{\prime}\right\}: U^{\prime} \in B_{r+s}\right\}$ for $s \in\{1, \ldots, k-r\}$, and $B_{k-r+1}^{r}=\left\{\left\{U: U \in B_{k}\right\}\right\}$. Notice that $\underline{B^{r}}=\underline{B}$ if $r=0$, whereas $\underline{B^{k}}$ is the trivial levels structure $\underline{B_{0}}$ on the player set $\left\{U: U \in B_{k}\right\}$. Notice as well that monotonicity of $(N, v)$ implies monotonicity of $\left(B_{r}, v^{r}\right)$.

On the other hand, given $\underline{B} \in \mathcal{L}(N)$ with $\underline{B}=\left\{B_{0}, \ldots, B_{k+1}\right\}$ and $i \in N$, let $\left(N, \underline{B^{-i}}\right) \in$ $\mathcal{L}(N)$ be the levels structure of cooperation obtained from $(N, \underline{B})$ by isolating player $i$ from 
the union she belongs to at each level, i.e., $\underline{B^{-i}}=\left\{B_{0}, B_{1}^{-i}, \ldots, B_{k}^{-i}, B_{k+1}\right\}$, where, for all $r \in\{1, \ldots, k\}, B_{r}^{-i}=\left\{U \in B_{r}: i \notin U\right\} \cup\{S \backslash i,\{i\}\}$ with $i \in S \in B_{r}$.

A value on $\mathcal{G L}$ is a map $f$ that assigns to every game with levels structure of cooperation $(N, v, \underline{B}) \in \mathcal{G L}$ a vector $f(N, v, \underline{B}) \in \mathbb{R}^{N}$. We recall the definition of the Shapley levels value (Winter, 1989) for games with levels structure of cooperation. To do so we need to introduce some further notation. For $\underline{B}=\left\{B_{0}, \ldots, B_{k+1}\right\} \in \mathcal{L}(N)$, we define the sets of permutations $\Omega_{1}(\underline{B}) \subseteq \Omega_{2}(\underline{B}) \subseteq \cdots \subseteq \Omega_{k+1}(\underline{B}) \subseteq \Pi(N)$ by $\Omega_{k+1}(\underline{B})=\Pi(N)$ and, recursively, for $r=$ $k, \ldots, 1$,

$$
\Omega_{r}(\underline{B})=\left\{\sigma \in \Omega_{r+1}(\underline{B}): \forall S \in B_{r}, \forall i, j \in S \text { and } l \in N \text {, if } \sigma(i)<\sigma(l)<\sigma(j) \text { then } l \in S\right\} .
$$

That is, $\Omega_{r}(\underline{B})$ denotes the permutations of $\Omega_{r+1}(\underline{B})$ such that the elements of each union of $B_{r}$ are consecutive.

\section{Definition 4.2. (Winter, 1989)}

The Shapley levels value, $\mathrm{Sh}^{\mathrm{L}}$, assigns to any cooperative game with levels structure of cooperation $(N, v, \underline{B}) \in \mathcal{G} \mathcal{L}$, a vector in $\mathbb{R}^{N}$ defined as

$$
\mathrm{Sh}_{i}^{\mathrm{L}}(N, v, \underline{B})=\frac{1}{\left|\Omega_{1}(\underline{B})\right|} \sum_{\sigma \in \Omega_{1}(\underline{B})}\left(v\left(P_{i}^{\sigma} \cup i\right)-v\left(P_{i}^{\sigma}\right)\right), i \in N
$$

where $P_{i}^{\sigma}=\{j \in N: \sigma(j)<\sigma(i)\}$ is the set of predecessors of $i$ with respect to $\sigma$.

\subsection{A class of LS-share functions}

In this section we introduce a family of share functions for games with levels structures of cooperation. The different share functions depend only on the choice of a positive, additive and anonymous real valued function $\mu: \mathcal{G} \rightarrow \mathbb{R}$. We generalize the families of share functions for standard games (van der Laan and van den Brink, 1998) and Coalition Structure (CS)-share functions for games with coalition structure (van den Brink and van der Laan, 2005) to games with an arbitrary number of levels structures of cooperation.

A Levels Structure (LS)-share function is a map that assigns to every player in a game with levels structure of cooperation $(N, v, \underline{B}) \in \mathcal{G} \mathcal{L}$ her share in the worth to be allocated over the players. The class of CS-share functions introduced and characterized in van den Brink and van der Laan (2005) is built by multiplying for each player two shares, namely the share of each player in some 'internal' game within the union she belongs to, and the share of this union in an 'external' game played among the unions. 
To generalize the class of CS-share functions to games with an arbitrary number of levels structure of cooperation $(N, v, \underline{B})$, we introduce further concepts and notation. Given a levels structure of cooperation $\underline{B} \in \mathcal{L}(N)$, for each player $i \in N$, let $U_{r}^{i}, r \in\{0, \ldots, k+1\}$, be the sequence of sets obtained by taking $U_{r}^{i}$ the element in $B_{r}$ that contains player $i$. Notice that $U_{0}^{i}=\{i\}, U_{k+1}^{i}=N$ and that $U_{0}^{i} \subseteq U_{1}^{i} \subseteq \cdots \subseteq U_{k}^{i} \subseteq U_{k+1}^{i}$. Then, let $N_{r}^{i}(\underline{B})=\{U: U \in$ $\left.B_{r}, U \subseteq U_{r+1}^{i}\right\}$ be the set of all unions of the $r^{t h}$-level of cooperation that form the union $U_{r+1}^{i}$ of the $(r+1)^{t h}$ level of cooperation. In order to ease the notation, we write $N_{r}^{i}$ when no confusion may arise.

Example 4.3 (Continuation of Example 4.1). Let $i=4 \in N$. Then,

$$
\begin{aligned}
& U_{0}^{4}=\{4\} \subseteq U_{1}^{4}=\{4,5\} \subseteq U_{2}^{4}=\{3,4,5\} \subseteq U_{3}^{4}=\{1,2,3,4,5\} \quad \text { and } \\
& N_{0}^{4}(\underline{B})=\{\{4\},\{5\}\}, N_{1}^{4}(\underline{B})=\{\{3\},\{4,5\}\}, N_{2}^{4}(\underline{B})=\{\{1,2\},\{3,4,5\}\} .
\end{aligned}
$$

Following the philosophy behind the definition of the internal and external games for games with coalition structure, given a game with $k$ levels structure of cooperation we shall define $k+1$ different games for each player. We recall that lowercase letters denote cardinalities, and hence in particular $n_{r}^{i}=\left|N_{r}^{i}\right|$ and $s_{r}=\left|S_{r}\right|$ for every $r \in\{0, \ldots k\}$ in the definition below.

Definition 4.4. Let $\mu: \mathcal{G} \rightarrow \mathbb{R}$ be positive, additive, and anonymous with corresponding weights $\left\{\omega_{\mu}^{n, s}: n \in \mathbb{N}, s \in\{0,1, \ldots, n-1\}\right\}$. For every $(N, v, \underline{B}) \in \mathcal{G} \mathcal{L}, i \in N$, and $r \in\{0, \ldots, k\}$, let the $r^{\text {th }}$-level game of $i$ with respect to $\mu,\left(N_{r}^{i}(\underline{B}), v_{\mu}^{i, r}\right) \in \mathcal{G}$, be given, for each $T \subseteq N_{r}^{i}$, by

$$
v_{\mu}^{i, r}(T)=\sum_{\substack{S_{k} \subseteq N_{k}^{i} \\ U_{k}^{i} \notin S_{k}}} \cdots \sum_{\substack{S_{r+1} \subseteq N_{r+1}^{i} \\ U_{r+1}^{i} \notin S_{r+1}}} \omega_{\mu}^{n_{k}^{i}, s_{k}} \cdots \omega_{\mu}^{n_{r+1}^{i}, s_{r+1}} \cdot\left[v\left(S_{k, r+1} \cup T\right)-v\left(S_{k, r+1}\right)\right]
$$

where $S_{k, r+1}=S_{k} \cup S_{k-1} \cup \cdots \cup S_{r+1}$ for $r \in\{0, \ldots, k-1\}$ and $S_{k, k+1}=\emptyset$.

Note that in the definition above we abuse notation and write $v\left(S_{k, r+1}\right)$ to denote $v(\{i \in$ $U: U \in S_{l}$ for some $\left.\left.l \in\{r+1, \ldots, k\}\right\}\right)$. Further, we assume that the empty set always belongs to the summation in eq. (8). Therefore the $k^{\text {th }}$ level game of any player with respect to any $\mu$ coincides with the $k^{t h}$ union level game, i.e., we have $v_{\mu}^{i, k}(T)=v\left(\bigcup_{U \in T} U\right)$ for all $T \subseteq N_{k}^{i}=\left\{U \in B_{k}: U \subseteq N\right\}=B_{k}$. Moreover, it is easy to check that, when $k=1, v_{\mu}^{i, 0}$ and $v_{\mu}^{i, 1}$ correspond exactly to the internal and external (quotient) games considered by Owen (1977) for $\mu=\mu^{\text {Sh }}$ and in Andjiga and Courtin (2010) for general $\mu$; in the latter paper a minor mistake in the internal game made in van den Brink and van der Laan (2005) has been corrected. It is as well straightforward to check that for every $i \in N$ and $r \in\{0, \ldots, k\},\left(N_{r}^{i}, v_{\mu}^{i, r}\right) \in \mathcal{G}$ whenever $(N, v) \in \mathcal{G}$. 
Example 4.5 (Continuation of Example 4.3). For $i=4, r=0$ and $T=\{4\}$, we have

$$
\begin{aligned}
v_{\mu}^{4,0}(\{4\}) & =\omega_{\mu}^{2,0} \cdot \omega_{\mu}^{2,0} \cdot(v(\{4\})-v(\emptyset))+\omega_{\mu}^{2,0} \cdot \omega_{\mu}^{2,1} \cdot(v(\{3,4\})-v(\{3\})) \\
& +\omega_{\mu}^{2,1} \cdot \omega_{\mu}^{2,0} \cdot(v(\{1,2,4\})-v(\{1,2\}))+\omega_{\mu}^{2,1} \cdot \omega_{\mu}^{2,1} \cdot(v(\{1,2,3,4\})-v(\{1,2,3\})) .
\end{aligned}
$$

Next, in line with Theorem 3.1 in van den Brink and van der Laan (2005), a class of LS-share functions is defined based on positive, additive, and anonymous $\mu$-functions. Each member of this class of LS-share functions is also denoted by $\rho^{\mu}$ and is defined as a product of the shares in each of the $r^{t h}$-level games.

Definition 4.6. Let $\mu: \mathcal{G} \rightarrow \mathbb{R}$ be positive, additive, and anonymous. Given a game with levels structure of cooperation $(N, v, \underline{B}) \in \mathcal{G} \mathcal{L}$, the $\mu$-LS-share function, $\rho^{\mu}$, assigns to any game $(N, v)$ a vector in $\mathbb{R}^{N}$ defined as

$$
\rho_{i}^{\mu}(N, v, \underline{B})=\prod_{r=0}^{k} \rho_{U_{r}^{i}}^{\mu}\left(N_{r}^{i}, v_{\mu}^{i, r}\right), i \in N,
$$

where the share function for standard games, $\rho^{\mu}$, is defined in Proposition 3.3.

It is easy to verify that the above definition indeed yields a LS-share function: this is just a consequence of $\rho^{\mu}$ being a share function and the fact that, for each $r \in\{1, \ldots, k\}$ and $U \in B_{r}$, it holds $N_{s}^{i}=N_{s}^{j}$ and $v_{\mu}^{i, r}=v_{\mu}^{j, r}$ for every pair of players $i, j \in U$ and $s \in\{r, \ldots, k\}$. The next proposition shows other properties satisfied by $\mu$-LS-share functions.

Proposition 4.7. Let $\mu: \mathcal{G} \rightarrow \mathbb{R}$ be positive, additive, and anonymous. Then

(i) For every $(N, v) \in \mathcal{G}, \rho^{\mu}\left(N, v, \underline{B_{0}}\right)=\rho^{\mu}(N, v)$.

(ii) For every $(N, v, \underline{B}) \in \mathcal{G} \mathcal{L}$ such that $(N, v) \in \mathcal{G}_{+}$, and every null player $i$ in $(N, v)$, $\rho_{i}^{\mu}(N, v, \underline{B})=0$.

(iii) For every $(N, v, \underline{B}) \in \mathcal{G L}$ and every pair of players $i, j \in N$ that are symmetric in $(N, v)$ such that there is some $U \in B_{1}$ with $i, j \in U, \rho_{i}^{\mu}(N, v, \underline{B})=\rho_{j}^{\mu}(N, v, \underline{B})$.

(iv) For every $(N, v, \underline{B}) \in \mathcal{G} \mathcal{L}, U \in B_{r} \in \underline{B}$ and $r \in\{0, \ldots, k\}, \sum_{i \in U} \rho_{i}^{\mu}(N, v, \underline{B})=\rho_{U}^{\mu}\left(B_{r}, v^{r}, \underline{B^{r}}\right)$.

(v) For every pair of games $(N, v),(N, w) \in \mathcal{G}$, every levels structure of cooperation $\underline{B} \in \mathcal{L}(N)$, and every player $i \in N$,

$\rho_{i}^{\mu}(N, z, \underline{B}) \cdot \prod_{r=0}^{k} \mu\left(N_{r}^{i}, z_{\mu}^{i, r}\right)=\prod_{r=0}^{k}\left[\mu\left(N_{r}^{i}, v_{\mu}^{i, r}\right) \rho_{U_{r}^{i}}^{\mu}\left(N_{r}^{i}, v_{\mu}^{i, r}, \underline{B_{0}}\right)+\mu\left(N_{r}^{i}, w_{\mu}^{i, r}\right) \rho_{U_{r}^{i}}^{\mu}\left(N_{r}^{i}, w_{\mu}^{i, r}, \underline{B_{0}}\right)\right]$, where $z=v+w$. 


\section{Proof.}

(i) If $\underline{B_{0}}=\left\{B_{0}, B_{1}\right\}$ then, for every $i \in N$, we have $N_{0}^{i}=\{\{j\}: j \in N\}$ and $N_{1}^{i}=\{N\}$. It trivially follows that $\rho^{\mu}\left(N, v, \underline{B_{0}}\right)=\rho_{U_{0}^{i}}^{\mu}\left(N_{0}^{i}, v_{\mu}^{i, 0}\right)=\rho_{i}^{\mu}(N, v)$.

(ii) We assume $k \geq 1$ since it is trivial otherwise. Let $(N, v, \underline{B}) \in \mathcal{G} \mathcal{L}$ be such that $(N, v) \in \mathcal{G}_{+}$, and $i \in N$ be a null player in $(N, v)$. First of all, observe that by Definition 4.4, $(N, v) \in \mathcal{G}_{+}$implies that $\left(N_{k}^{i}, v_{\mu}^{i, k}\right) \in \mathcal{G}_{+}$. We next shall show that, for every $r \in\{1, \ldots, k\}$, if $U_{r}^{i}$ is not a null player in $\left(N_{r}^{i}, v_{\mu}^{i, r}\right)$, then $\left(N_{r-1}^{i}, v_{\mu}^{i, r-1}\right) \in \mathcal{G}_{+}$.

We first show it for $r=k$. Let $U_{k}^{i} \in N_{k}^{i}$ be a non-null player in $\left(N_{k}^{i}, v_{\mu}^{i, k}\right)$. That is, there is $S_{k}^{*} \subseteq N_{k}^{i} \backslash U_{k}^{i}$ such that

$$
v_{\mu}^{i, k}\left(S_{k}^{*} \cup U_{k}^{i}\right)-v_{\mu}^{i, k}\left(S_{k}^{*}\right)=v\left(S_{k}^{*} \cup U_{k}^{i}\right)-v\left(S_{k}^{*}\right)>0 .
$$

Next, we show that $\left(N_{k-1}^{i}, v_{\mu}^{i, k-1}\right)$ is not a null game. Indeed, note that

$$
v_{\mu}^{i, k-1}\left(N_{k-1}^{i}\right)=\sum_{\substack{S_{k} \subseteq N_{k}^{i} \\ U_{k}^{i} \notin S_{k}}} \omega_{\mu}^{n_{k}^{i}, s_{k}}\left[v\left(S_{k} \cup U_{k}^{i}\right)-v\left(S_{k}\right)\right]>0,
$$

where the inequality holds since, on the one hand, each term in the above summation is nonnegative because $(N, v)$ is monotone and, on the other hand, the term associated to $S_{k}^{*}$ is positive by eq. (9).

Now take $r \in\{1, \ldots, k-1\}$ and let $U_{r}^{i}$ be a non-null player in $\left(N_{r}^{i}, v_{\mu}^{i, r}\right)$. That is, there is $S_{r}^{*} \subseteq N_{r}^{i} \backslash U_{r}^{i}$ such that

$$
\begin{aligned}
& v_{\mu}^{i, r}\left(S_{r}^{*} \cup U_{r}^{i}\right)-v_{\mu}^{i, r}\left(S_{r}^{*}\right) \\
& =\sum_{\substack{S_{k} \subseteq N_{k}^{i} \\
U_{k}^{i} \notin S_{k}}} \cdots \sum_{\substack{S_{r+1} \subseteq N_{r+1}^{i} \\
U_{r+1}^{i} \notin S_{r+1}}} \omega_{\mu}^{n_{k}^{i}, s_{k}} \cdots \omega_{\mu}^{n_{r+1}^{i}, s_{r+1}}\left[v\left(S_{k, r+1} \cup S_{r}^{*} \cup U_{r}^{i}\right)-v\left(S_{k, r+1} \cup S_{r}^{*}\right)\right]>0 .
\end{aligned}
$$

Since $(N, v)$ is monotone, there are $S_{k}^{*} \subseteq N_{k}^{i} \backslash U_{k}^{i}, \ldots, S_{r+1}^{*} \subseteq N_{r+1}^{i} \backslash U_{r+1}^{i}$ such that

$$
v\left(S_{k, r}^{*} \cup U_{r}^{i}\right)-v\left(S_{k, r}^{*}\right)>0,
$$

where $S_{k, r}^{*}=S_{k}^{*} \cup \ldots \cup S_{r+1}^{*} \cup S_{r}^{*}$. Next, we show that $\left(N_{r-1}^{i}, v_{\mu}^{i, r-1}\right)$ is not a null game. Indeed, note that

$$
v_{\mu}^{i, r-1}\left(N_{r-1}^{i}\right)=\sum_{\substack{S_{k} \subseteq N_{k}^{i} \\ U_{k}^{i} \notin S_{k}}} \cdots \sum_{\substack{S_{r} \subseteq N_{r}^{i} \\ U_{r}^{i} \notin S_{r}}} \omega_{\mu}^{n_{k}^{i}, s_{k}} \cdots \omega_{\mu}^{n_{r}^{i}, s_{r}}\left[v\left(S_{k, r} \cup U_{r}^{i}\right)-v\left(S_{k, r}\right)\right]>0,
$$


where, again, the inequality holds since, on the one hand, each term in the above summation is nonnegative because $(N, v)$ is monotone and, on the other hand, the term associated to $S_{k, r}^{*}$ is positive by eq. (10).

Summing up, first we have seen that $\left(N_{k}^{i}, v_{\mu}^{i, k}\right)$ is not a null game, and second that, for every $r \in\{1, \ldots, k\}$, if $U_{r}^{i}$ is not a null player in $\left(N_{r}^{i}, v_{\mu}^{i, k}\right)$, then $\left(N_{r-1}^{i}, v_{\mu}^{i, r-1}\right) \in \mathcal{G}_{+}$. Thus, two cases may arise,

- either there exists $r \in\{1, \ldots, k\}$ such that $U_{r}^{i}$ is a null player in a non-null game $\left(N_{r}^{i}, v_{\mu}^{i, r}\right)$, which by NPP of $\rho^{\mu}$ means that $\rho_{U_{r}^{i}}^{\mu}\left(N_{r}^{i}, v_{\mu}^{i, r}\right)=0$,

- or, for every $r \in\{1, \ldots, k\},\left(N_{r-1}^{i}, v_{\mu}^{i, r-1}\right)$ is not a null game. In particular $\left(N_{0}^{i}, v_{\mu}^{i, 0}\right) \in \mathcal{G}_{+}$. Since $i$ is a null player in $(N, v)$ it is also a null player in $\left(N_{0}^{i}, v_{\mu}^{i, 0}\right)$, which by NPP of $\rho^{\mu}$ means that $\rho_{U_{0}^{i}}^{\mu}\left(N_{0}^{i}, v_{\mu}^{i, 0}\right)=0$.

In any case, we obtain $\rho_{i}^{\mu}(N, v, \underline{B})=0$.

(iii) Notice that, if $i$ and $j$ are symmetric players in $(N, v)$ and they belong to the same union at the first level, then $U_{0}^{i}$ and $U_{0}^{j}$ are symmetric in $\left(N_{0}^{i}, v_{\mu}^{i, 0}\right)=\left(N_{0}^{j}, v_{\mu}^{j, 0}\right)$ and $U_{r}^{i}=U_{r}^{j}$ for $r \in\{1, \ldots, k\}$. Then, using Definition 4.6 and the fact that $\rho^{\mu}$ satisfies SYM,

$$
\rho_{i}^{\mu}(N, v, \underline{B})=\rho_{U_{0}^{i}}^{\mu}\left(N_{0}^{i}, v_{\mu}^{i, 0}\right) \prod_{r=1}^{k} \rho_{U_{r}^{i}}^{\mu}\left(N_{r}^{i}, v_{\mu}^{i, r}\right)=\rho_{U_{0}^{j}}^{\mu}\left(N_{0}^{j}, v_{\mu}^{j, 0}\right) \prod_{r=1}^{k} \rho_{U_{r}^{j}}^{\mu}\left(N_{r}^{j}, v_{\mu}^{j, r}\right)=\rho_{j}^{\mu}(N, v, \underline{B}) .
$$

(iv) This is a consequence of $\rho^{\mu}$ being a share function and the fact that given $U \in B_{r} \in \underline{B}$, for every $l \in\{r, \ldots, k\},\left(N_{l}^{i}, v_{\mu}^{i, l}\right)$ is the same game for any $i \in U$.

(v) This follows from Proposition 3.3 and the definition of $\rho^{\mu}$ since, for each $r \in\{0, \ldots, k\}$ and each $i \in N$, it holds that $(v+w)_{\mu}^{i, r}=v_{\mu}^{i, r}+w_{\mu}^{i, r}$.

Property (i) shows that the $\mu$-LS-share function extends the $\mu$-share function for games without levels structure of cooperation. Properties (ii) and (iii) are standard and apply to every game with levels structure of cooperation. Although only weaker versions of these two properties are needed in the characterization results for $\rho^{\mu}$ in Section 5 , it is important to point out that their corresponding stronger versions are also satisfied by $\rho^{\mu}$. In fact, van den Brink and van der Laan (2005) use, for games with one level structure of cooperation, the stronger versions. Property (iv) is a consistency property, in the sense that it requires the share of a union in the $r^{t h}$ - union level game to coincide with the sum of the shares of the players in that union. For 
games with coalition structure it generalizes the quotient game property stated in Owen (1977). Lastly, property (v) is a generalized version of the $\mu$-ADD for share functions and it extends as well two $\mu$-additivity properties introduced in van den Brink and van der Laan (2005) for CS-share functions.

\subsection{The Shapley levels share function}

The LS-share function associated to the Shapley levels value as given in Definition 4.2 is defined below. Since $\mathrm{Sh}^{\mathrm{L}}$ is efficient, we only need to divide the Shapley value of a player by the worth of the grand coalition.

Definition 4.8. Given a game with levels structure of cooperation $(N, v, \underline{B}) \in \mathcal{G} \mathcal{L}$, the Shapley levels $L S$-share function, $\rho^{\mathrm{Sh}^{\mathrm{L}}}$, is a vector in $\mathbb{R}^{N}$ given by,

$$
\rho_{i}^{\mathrm{Sh}^{\mathrm{L}}}(N, v, \underline{B})=\frac{\mathrm{Sh}_{i}^{\mathrm{L}}(N, v, \underline{B})}{v(N)}, i \in N, \text { if } v \neq v_{0},
$$

and $\rho_{i}^{S h^{\mathrm{L}}}\left(N, v_{0}, \underline{B}\right)=\prod_{r=0}^{k} \frac{1}{\left|N_{r}^{i}\right|}, i \in N$.

In this subsection we prove that the Shapley levels LS-share function belongs to the family of share functions introduced in Definition 4.6. In order to do so, we make use of the characterization of the Shapley levels value by Álvarez-Mozos and Tejada (2011). The two main properties of this characterization are the Level Game Property and the Level Balanced Contributions property. The first one requires the total payoff obtained by the members of a union in a given level be equal to the payoff obtained by the union when considering it as a player in the corresponding level game. The second one is a reciprocity property that requires that, when a player of a union on the highest level $B_{k}$ is isolated within the level structure and becomes a union itself of $B_{k}, B_{k-1}, \ldots, B_{1}$, this affects a player that is in the same union on the lowest level $B_{1}$ in the same amount as if it happens the other way around. Let us formally introduce these two properties.

LGP A value $f$ on $\mathcal{G L}$ satisfies the Level Game Property if for every $(N, v, \underline{B}) \in \mathcal{G L}$ and $U \in B_{r}$ for some $r \in\{1, \cdots, k\}$,

$$
\sum_{i \in U} f_{i}(N, v, \underline{B})=f_{U}\left(B_{r}, v^{r}, \underline{B_{r}}\right) .
$$

LBC A value $f$ on $\mathcal{G L}$ satisfies Level Balanced Contributions if for every $(N, v, \underline{B}) \in \mathcal{G L}$ and $i, j \in U \in B_{1}$,

$$
f_{i}(N, v, \underline{B})-f_{i}\left(N, v, \underline{B^{-j}}\right)=f_{j}(N, v, \underline{B})-f_{j}\left(N, v, \underline{B^{-i}}\right) .
$$


Before proving the main result of this subsection, we first state a useful alternative expression of the Shapley levels value.

Lemma 4.9. Let $(N, v, \underline{B}) \in \mathcal{G L}$ be a game with levels structure of cooperation. Then, for each $i \in N$,

$$
\mathrm{Sh}_{i}^{\mathrm{L}}(N, v, \underline{B})=\sum_{S_{k} \subseteq N_{k}^{i} \backslash U_{k}^{i}} \cdots \sum_{S_{0} \subseteq N_{0}^{i} \backslash U_{0}^{i}} \omega_{\mathrm{Sh}}^{n_{k}^{i}, s_{k}} \cdots \omega_{\mathrm{Sh}}^{n_{0}^{i}, s_{0}} \cdot\left[v\left(S_{k, 0} \cup i\right)-v\left(S_{k, 0}\right)\right] .
$$

Proof. We prove this lemma by induction on the number of levels of $\underline{B} \in \mathcal{L}(N)$. The case $k=1$ is a consequence of $\mathrm{Sh}^{\mathrm{L}}$ being a generalization of the Owen value. Suppose that the Shapley levels value $\operatorname{Sh}^{\mathrm{L}}\left(N, v, \underline{B}^{\prime}\right)$ is obtained from eq. (11) for every $\left(N^{\prime}, v^{\prime}, \underline{B^{\prime}}\right) \in \mathcal{G L}$ with at most $k-1$ levels of cooperation, and let $(N, v, \underline{B}) \in \mathcal{G L}$ be a game with $k$ levels of cooperation. Let $i \in U_{1}^{i} \in B_{1}$ be an arbitrary player. We prove that $\operatorname{Sh}_{i}^{\mathrm{L}}(N, v, \underline{B})$ is obtained from eq. (11) for $(N, v, \underline{B})$ by a second induction on $u=\left|U_{1}^{i}\right|$. If $u=1$, we have $U_{1}^{i}=\{i\}$ and thus

$$
\begin{aligned}
\operatorname{Sh}_{i}^{\mathrm{L}}(N, v, \underline{B})= & \sum_{i \in U_{1}^{i}} \operatorname{Sh}_{i}^{\mathrm{L}}(N, v, \underline{B})=\operatorname{Sh}_{\{i\}}^{\mathrm{L}}\left(B_{1}, v^{1}, \underline{B_{1}}\right) \\
= & \sum_{\substack{S_{k} \subseteq N_{k}^{\{i\}}\left(\underline{B_{1}}\right) \\
U_{k}^{\{i\}}\left(\underline{B_{1}}\right) \notin S_{k}}} \cdots \sum_{\substack{S_{1} \subseteq N_{1}^{\{i\}}\left(\underline{B_{1}}\right) \\
U_{1}^{\{i\}}\left(\underline{B_{1}}\right) \notin S_{1}}} \omega_{\mathrm{Sh}}^{n_{k}^{i}\left(\underline{B_{1}}\right), s_{k}} \cdots \omega_{\mathrm{Sh}}^{n_{1}^{i}\left(\underline{B_{1}}\right), s_{1}}\left[v^{1}\left(S_{k, 1} \cup\{i\}\right)-v^{1}\left(S_{k, 1}\right)\right] \\
= & \sum_{\substack{S_{k} \subseteq N_{k}^{i} \\
U_{k}^{i} \notin S_{k}}} \cdots \sum_{\substack{S_{1} \subseteq N_{1}^{i} \\
U_{1}^{i} \notin S_{1}}} \sum_{S_{0}^{i} \notin N_{0}^{i}} \omega_{\mathrm{Sh}}^{n_{k}^{i}, s_{k}} \cdots \omega_{\mathrm{Sh}}^{n_{0}^{i}, s_{0}}\left[v\left(S_{k, 0} \cup i\right)-v\left(S_{k, 0}\right)\right],
\end{aligned}
$$

where the second equality follows by LGP, the third equality holds by the first induction hypothesis since $\underline{B_{1}} \in \mathcal{L}\left(B_{1}\right)$ is a levels structure of cooperation with $k-1$ levels, and the fourth equality holds since $N_{0}^{i}(\underline{B}) \backslash U_{0}^{i}=\emptyset, N_{r}^{i}\left(B_{1}, \underline{B_{1}}\right)=N_{r}^{i}(\underline{B})$ for all $r \in\{1, \ldots, k\}$, and $v^{1}\left(S_{k, 1} \cup\{i\}\right)-v^{1}\left(S_{k, 1}\right)=v\left(S_{k, 0} \cup i\right)-v\left(S_{k, 0}\right)$.

Now assume that $\operatorname{Sh}_{i}^{\mathrm{L}}(N, v, \underline{B})$ is obtained from eq. (11) for any $(N, v, \underline{B})$ with $k$ levels of cooperation and for any $i \in U_{1}^{i} \in B_{1}$ where $\left|U_{1}^{i}\right|<u$. Next suppose that $(N, v, \underline{B}) \in \mathcal{G L}$ is a game with $k$ levels of cooperation and $\left|U_{1}^{i}\right|=u$. Since $\mathrm{Sh}^{\mathrm{L}}$ satisfies LGP and LBC, we have

$$
\begin{aligned}
\operatorname{Sh}_{i}^{\mathrm{L}}(N, v, \underline{B}) & =\mathrm{Sh}_{U_{1}^{i}}^{\mathrm{L}}\left(B_{1}, v^{1}, \underline{B_{1}}\right)-\sum_{j \in U_{1}^{i} \backslash i} \operatorname{Sh}_{j}^{\mathrm{L}}(N, v, \underline{B}) \\
& =\mathrm{Sh}_{U_{1}^{i}}^{\mathrm{L}}\left(B_{1}, v^{1}, \underline{B_{1}}\right)-\sum_{j \in U_{1}^{i} \backslash i}\left[\operatorname{Sh}_{i}^{\mathrm{L}}(N, v, \underline{B})-\mathrm{Sh}_{i}^{\mathrm{L}}\left(N, v, \underline{B^{-j}}\right)+\mathrm{Sh}_{j}^{\mathrm{L}}\left(N, v, \underline{B^{-i}}\right)\right],
\end{aligned}
$$

which can be rewritten as

$$
\operatorname{Sh}_{i}^{\mathrm{L}}(N, v, \underline{B})=\frac{1}{n_{0}^{i}}\left(\operatorname{Sh}_{U_{1}^{i}}^{\mathrm{L}}\left(B_{1}, v^{1}, \underline{B_{1}}\right)+\sum_{j \in U_{1}^{i} \backslash i}\left[\operatorname{Sh}_{i}^{\mathrm{L}}\left(N, v, \underline{B^{-j}}\right)-\operatorname{Sh}_{j}^{\mathrm{L}}\left(N, v, \underline{B^{-i}}\right)\right]\right) .
$$


Observe that, according to the double induction hypothesis, $\mathrm{Sh}_{U_{1}^{i}}^{\mathrm{L}}\left(B_{1}, v^{1}, \underline{B_{1}}\right), \mathrm{Sh}_{i}^{\mathrm{L}}\left(N, v, \underline{B^{-j}}\right)$, and $\operatorname{Sh}_{j}^{\mathrm{L}}\left(N, v, \underline{B^{-i}}\right)$, for all $j \in U_{1}^{i} \backslash i$, can be obtained from eq. (11). Hence, for every $j \in U_{1}^{i} \backslash i$, it holds that

$$
\begin{aligned}
& \operatorname{Sh}_{i}^{\mathrm{L}}\left(N, v, \underline{B^{-j}}\right)=\sum_{\substack{S_{k} \subseteq N_{k}^{i}\left(\underline{B^{-j}}\right) \\
U_{k}^{i}\left(\underline{B^{-j}}\right) \notin S_{k}}} \cdots \sum_{\substack{S_{0} \subseteq N_{0}^{i}\left(\underline{B^{-j}}\right) \\
U_{0}^{i}\left(\underline{B^{-j}}\right) \notin S_{0}}} \omega_{\mathrm{Sh}}^{n_{k}^{i}\left(\underline{B^{-j}}\right), s_{k}} \cdots \omega_{\mathrm{Sh}}^{n_{0}^{i}\left(B^{-j}\right), s_{0}}\left[v\left(S_{k, 0} \cup i\right)-\left(v\left(S_{k, 0}\right)\right]\right. \\
& =\sum_{\substack{S_{k} \subseteq N_{k}^{i}\left(\frac{B^{-j}}{2}\right) \\
\{j\}, U_{k}^{i}(\underline{\underline{B}}) \notin S_{k}}} \cdots \sum_{\substack{S_{0} \subseteq N_{0}^{i}\left(\underline{B^{-j}}\right) \\
U_{0}^{i}\left(\underline{B^{-j}}\right) \notin S_{0}}}\left\{\omega_{\mathrm{Sh}}^{n_{k}^{i}\left(\underline{B^{-j}}\right), s_{k}} \cdots \omega_{\mathrm{Sh}}^{n_{0}^{i}\left(\underline{B^{-j}}\right), s_{0}}\left[v\left(S_{k, 0} \cup i\right)-v\left(S_{k, 0}\right)\right]\right. \\
& \left.+\frac{s_{k}+1}{n_{k}^{i}\left(\underline{B^{-j}}\right)-s_{k}-1} \cdot \omega_{\mathrm{Sh}}^{n_{k}^{i}\left(\underline{B^{-j}}\right), s_{k}} \cdots \omega_{\mathrm{Sh}}^{n_{0}^{i}\left(\underline{B^{-j}}\right), s_{0}}\left[v\left(S_{k, 0} \cup j \cup i\right)-v\left(S_{k, 0} \cup j\right)\right]\right\} \text {, }
\end{aligned}
$$

where the second equality is obtained by distinguishing the cases $\{j\} \notin S_{k}$ and $\{j\} \in S_{k}$ and noting that

$$
\frac{\omega_{\mathrm{Sh}}^{n_{k}^{i}\left(\underline{B^{-j}}\right), s_{k}+1}}{\omega_{\mathrm{Sh}}^{n_{k}^{i}\left(\underline{B^{-j}}\right), s_{k}}}=\frac{s_{k}+1}{n_{k}^{i}\left(\underline{B^{-j}}\right)-s_{k}-1} .
$$

We point out that we have used the notation $U_{r}^{i}\left(\underline{B^{-j}}\right)$ for all $r \in\{0, \ldots, k+1\}$ to denote the unions of each level that contain player $i$ when the levels structure is $\underline{B^{-j}}$. Thus, $U_{0}^{i}\left(\underline{B^{-j}}\right)=U_{0}^{i}=\{i\}$ and $U_{r}^{i}\left(\underline{B^{-j}}\right)=U_{r}^{i} \backslash j$ for all $r \in\{1, \ldots, k\}$. Also notice that $N_{k}^{i}\left(\underline{B^{-j}}\right)=N_{k}^{i} \backslash U_{k}^{i} \cup\left(U_{k}^{i} \backslash j\right) \cup\{j\}$, $N_{r}^{i}\left(\underline{B^{-j}}\right)=N_{r}^{i} \backslash U_{r}^{i} \cup\left(U_{r}^{i} \backslash j\right)$ for each $r \in\{1, \ldots k-1\}$ and $N_{0}^{i}\left(\underline{B^{-j}}\right)=N_{0}^{i} \backslash j$. Moreover, it can also be checked that

$$
\begin{aligned}
& \frac{\left.\omega_{\mathrm{Sh}}^{n_{k}^{i}\left(B^{-j}\right.}\right), s_{k}}{\omega_{\mathrm{Sh}}^{n_{k}^{i}, s_{k}}}=\frac{n_{i}^{k}-s_{k}}{n_{i}^{k}+1}, \quad \quad \frac{\left.\omega_{\mathrm{Sh}}^{n_{0}^{i}\left(B^{-j}\right.}\right), s_{0}}{\omega_{\mathrm{Sh}}^{n_{0}^{i}, s_{0}}}=\frac{n_{0}^{i}}{n_{0}^{i}-s_{0}+1}, \quad \text { and } \\
& \frac{\left.\omega_{\mathrm{Sh}}^{n_{r}^{i}\left(B^{-j}\right.}\right), s_{r}}{\omega_{\mathrm{Sh}}^{n_{r}^{i}, s_{r}}}=1 \text {, for every } r \in\{1, \ldots, k-1\} .
\end{aligned}
$$

From the above remarks we obtain

$$
\begin{aligned}
& \operatorname{Sh}_{i}^{\mathrm{L}}\left(N, v, \underline{B^{-j}}\right) \\
& =\sum_{\substack{S_{k} \subseteq N_{k}^{i} \\
U_{k}^{i} \notin S_{k}}} \cdots \sum_{\substack{S_{0} \subseteq N_{0}^{i} \\
\{i\},\{j\} \notin S_{0}}}\left\{\left(\frac{n_{k}^{i}-s_{k}}{n_{k}^{i}+1} \cdot \frac{n_{0}^{i}}{n_{0}^{i}-s_{0}-1}\right) \cdot \omega_{\mathrm{Sh}}^{n_{k}^{i}, s_{k}} \cdots \omega_{\mathrm{Sh}}^{n_{0}^{i}, s_{0}}\left[v\left(S_{k, 0} \cup i\right)-v\left(S_{k, 0}\right)\right]\right. \\
& \left.+\left(\frac{s_{k}+1}{n_{k}^{i}+1} \cdot \frac{n_{0}^{i}}{n_{0}^{i}-s_{0}-1}\right) \cdot \omega_{\mathrm{Sh}}^{n_{k}^{i}, s_{k}} \cdots \omega_{\mathrm{Sh}}^{n_{0}^{i}, s_{0}}\left[v\left(S_{k, 0} \cup j \cup i\right)-v\left(S_{k, 0} \cup j\right)\right]\right\}
\end{aligned}
$$


Using twice the above expression, exchanging $i$ and $j$, we obtain,

$$
\begin{aligned}
& \operatorname{Sh}_{i}^{\mathrm{L}}\left(N, v, \underline{B^{-j}}\right)-\operatorname{Sh}_{j}^{\mathrm{L}}\left(N, v, \underline{B^{-i}}\right) \\
& =\sum_{\substack{S_{k} \subseteq N_{k}^{i} \\
U_{k}^{i} \notin S_{k}}} \cdots \sum_{\substack{S_{0} \subseteq N_{0}^{i} \\
\{i\},\{j\} \notin S_{0}}} \frac{n_{0}^{i}}{n_{0}^{i}-s_{0}-1} \cdot \omega_{\mathrm{Sh}}^{n_{k}^{i}, s_{k}} \cdots \omega_{\mathrm{Sh}}^{n_{0}^{i}, s_{0}}\left[v\left(S_{k, 0} \cup i\right)-v\left(S_{k, 0} \cup j\right)\right] .
\end{aligned}
$$

Then, from eq. (12), we have

$$
\begin{aligned}
& \operatorname{Sh}_{i}^{\mathrm{L}}(N, v, \underline{B})=\frac{1}{n_{0}^{i}} \sum_{\substack{S_{k} \subseteq N_{k}^{i} \\
U_{k}^{i} \notin S_{k}}} \cdots \sum_{\substack{S_{1} \subseteq N_{1}^{i} \\
U_{1}^{i} \notin S_{1}}} \omega_{\mathrm{Sh}}^{n_{k}^{i}, s_{k}} \cdots \omega_{\mathrm{Sh}}^{n_{1}^{i}, s_{1}} \\
& \cdot\left\{\begin{array}{c}
\left.v\left(S_{k, 1} \cup U_{1}^{i}\right)-v\left(S_{k, 1}\right)+\sum_{j \in U_{1}^{i} \backslash i} \sum_{\substack{S_{0} \subseteq N_{0}^{i} \\
\{i\},\{j\} \notin S_{0}}} \frac{n_{0}^{i}}{n_{0}^{i}-s_{0}-1} \cdot \omega_{\mathrm{Sh}}^{n_{0}^{i}, s_{0}}\left[v\left(S_{k, 0} \cup i\right)-v\left(S_{k, 0} \cup j\right)\right]\right\} .
\end{array}\right.
\end{aligned}
$$

Lastly, the expression of the lemma follows since, given $S_{k} \subseteq N_{k}^{i} \backslash U_{k}^{i}, \ldots, S_{1} \subseteq N_{1}^{i} \backslash U_{1}^{i}$, we have

$$
\begin{aligned}
& \frac{1}{n_{0}^{i}}\left[v\left(S_{k, 1} \cup U_{1}^{i}\right)-v\left(S_{k, 1}\right)\right]+\sum_{j \in U_{1}^{i} \backslash i} \sum_{\substack{S_{0} \subseteq N_{0}^{i} \\
\{i\},\{j\} \notin S_{0}}} \frac{1}{n_{0}^{i}-s_{0}-1} \cdot \omega_{\mathrm{Sh}}^{n_{0}^{i}, s_{0}} \cdot\left[v\left(S_{k, 0} \cup i\right)-v\left(S_{k, 0} \cup j\right)\right] \\
& =\frac{1}{n_{0}^{i}}\left[v\left(S_{k, 1} \cup U_{1}^{i}\right)-v\left(S_{k, 1}\right)\right]+\sum_{\substack{S_{0} \subseteq N_{0}^{i} \backslash\{i\} \\
S_{0} \neq N_{0}^{i} \backslash\{i\}}}\left(n_{0}^{i}-s_{0}-1\right) \cdot \frac{1}{n_{0}^{i}-s_{0}-1} \cdot \omega_{\mathrm{Sh}}^{n_{0}^{i}, s_{0}} \cdot v\left(S_{k, 0} \cup i\right) \\
& -\sum_{\substack{S_{0} \subseteq N_{0}^{i} \backslash\{i\} \\
S_{0} \neq \emptyset}} s_{0} \cdot \frac{1}{n_{0}^{i}-s_{0}} \cdot \omega_{\mathrm{Sh}}^{n_{0}^{i}, s_{0}-1} \cdot v\left(S_{k, 0}\right)=\sum_{S_{0} \subseteq N_{0}^{i} \backslash U_{0}^{i}} \omega_{\mathrm{Sh}}^{n_{0}^{i}, s_{0}}\left[v\left(S_{k, 0} \cup i\right)-v\left(S_{k, 0}\right)\right] \text {, }
\end{aligned}
$$

where the first equality holds by observing that, given $S_{0} \subsetneq N_{0}^{i} \backslash\{i\}$, the number of different players $j \in N_{0}^{i} \backslash\{i\}$ such that $j \notin S_{0}$ is $n_{0}^{i}-s_{0}-1$, whereas, given $\emptyset \neq S_{0} \subseteq N_{0}^{i} \backslash\{i\}$, the number of different players $j \in N_{0}^{i} \backslash\{i\}$ such that $j \in S_{0}$ is $s_{0}$.

We now prove that the Shapley levels LS-share function, $\rho^{S \mathrm{~h}^{\mathrm{L}}}$, lies within the class of $\mu$-LS-share functions, i.e., that there is an anonymous, positive, and additive real-valued $\mu$ such that $\rho^{\mathrm{Sh}^{\mathrm{L}}}=\rho^{\mu}$. Furthermore, we prove that $\mu=\mu^{\mathrm{Sh}}$ and that the corresponding weights are therefore those given in eq. (7).

Proposition 4.10. Let $(N, v, \underline{B}) \in \mathcal{G L}$ be a game with levels structure of cooperation. Then

$$
\rho^{\mathrm{Sh}^{\mathrm{L}}}(N, v, \underline{B})=\rho^{\mu^{\mathrm{Sh}}}(N, v, \underline{B}) .
$$

Proof. Let $(N, v, \underline{B}) \in \mathcal{G L}, i \in N$, and $\mu=\mu^{\text {Sh }}$. First of all, note that if $(N, v)$ is a null game the result is straightforward. Then, in the sequel we assume that $(N, v) \in \mathcal{G}_{+}$. If $i$ is a 
null player in $(N, v)$, by NPP, we have $\rho_{i}^{S h^{\mathrm{L}}}(N, v, \underline{B})=0$, whereas, by (ii) of Proposition 4.7, we have $\rho_{i}^{\mu^{\text {sh }}}(N, v, \underline{B})=0$, and thus eq. (13) trivially holds. Thus, we also assume that $i$ is not a null player.

From the proof of (ii) in Proposition 4.7, we know that, for every $r \in\{1, \ldots, k\}$, if $U_{r}^{i}$ is not a null player in $\left(N_{r}^{i}, v_{\mu}^{i, r}\right)$ then $\left(N_{r-1}^{i}, v_{\mu}^{i, r-1}\right)$ is not a null game, and that $\left(N_{k}^{i}, v_{\mu}^{i, k}\right)$ is not a null game when $(N, v)$ is not a null game either. Hence, if, for some $r \in\{1, \ldots, k\}, U_{r}^{i}$ is a null player in $\left(N_{r}^{i}, v_{\mu}^{i, r}\right)$, then this latter game is non-null.

We now consider two cases. First, suppose that, for some $r \in\{1, \ldots, k\}, U_{r}^{i}$ is a null player in $\left(N_{r}^{i}, v_{\mu}^{i, r}\right) \in \mathcal{G}_{+}$. First, by NPP we have that $\rho_{U_{r}^{i}}^{\mu}\left(N_{r}^{i}, v_{\mu}^{r, i}\right)=0$ and, hence, $\rho_{i}^{\mu}(N, v, \underline{B})=0$. Then, for every $S_{r} \subseteq N_{r}^{i} \backslash U_{r}^{i}$, we have $v_{\mu}^{i, r}\left(S_{r} \cup U_{r}^{i}\right)=v_{\mu}^{i, r}\left(S_{r}\right)$, which implies that $U_{r}^{i}$ is a null player also in $\left(B_{r}, v^{r}\right)$. Moreover, since $\mathrm{Sh}^{\mathrm{L}}$ satisfies the null player property, we obtain

$$
\mathrm{Sh}_{U_{r}^{i}}^{\mathrm{L}}\left(B_{r}, v^{r}, \underline{B_{r}}\right)=0 .
$$

Now, taking into account that $(N, v) \in \mathcal{G}_{+}$, we have that, for every $j \in N, \operatorname{Sh}_{j}^{\mathrm{L}}(N, v, \underline{B}) \geq 0$. Thus, from eq. (14) and the fact that $\mathrm{Sh}^{\mathrm{L}}$ satisfies LGP, we obtain that $\mathrm{Sh}_{i}^{\mathrm{L}}(N, v, \underline{B})=0$ and, hence, $\rho_{i}^{\mathrm{Sh}^{\mathrm{L}}}(N, v, \underline{B})=0$, which completes the proof for this case.

Second, assume that for every $r \in\{0, \ldots, k\},\left(N_{r}^{i}, v_{\mu}^{r, i}\right) \in \mathcal{G}_{+}$. Using Definition 4.6 and Proposition 3.3, it is enough to check that

$$
\rho_{i}^{\mathrm{Sh}^{\mathrm{L}}}(N, v, \underline{B})=\prod_{r=0}^{k} \frac{\mathrm{Sh}_{U_{r}^{i}}\left(N_{r}^{i}, v_{\mu}^{i, r}\right)}{v_{\mu}^{i, r}\left(N_{r}^{i}\right)} .
$$

First, note that by Definitions 2.1 and 4.4 , for each $r \in\{0, \ldots, k-1\}$,

$$
\mathrm{Sh}_{U_{r+1}^{i}}\left(N_{r+1}^{i}, v_{\mu}^{i, r+1}\right)=v_{\mu}^{i, r}\left(N_{r}^{i}\right) .
$$

Second, using Lemma 4.9, we have

$$
\begin{aligned}
\operatorname{Sh}_{i}^{\mathrm{L}}(N, v, \underline{B}) & =\sum_{S_{k} \subseteq N_{k}^{i} \backslash U_{k}^{i}} \cdots \sum_{S_{0} \subseteq N_{0}^{i} \backslash U_{0}^{i}} \omega_{\mathrm{Sh}}^{n_{k}^{i}, s_{k}} \cdots \omega_{\mathrm{Sh}}^{n_{0}^{i}, s_{0}}\left[v\left(S_{k, 0} \cup i\right)-v\left(S_{k, 0}\right)\right] \\
& =\sum_{\substack{S_{0} \subseteq N_{0}^{i} \\
U_{0}^{i} \notin S_{0}}} \omega_{\mathrm{Sh}}^{n_{0}^{i}, s_{0}}\left\{\sum_{\substack{S_{k} \subseteq N_{k}^{i} \\
U_{k}^{i} \notin S_{k}}} \cdots \sum_{\substack{S_{1} \subseteq N_{1}^{i} \\
U_{1}^{i} \notin S_{1}}} \omega_{\mathrm{Sh}}^{n_{k}^{i}, s_{k}} \cdots \omega_{\mathrm{Sh}}^{n_{1}^{i}, s_{1}}\left[v\left(S_{k, 0} \cup i\right)-v\left(S_{k, 0}\right)\right]\right\} \\
& =\operatorname{Sh}_{i}\left(N_{0}^{i}, v_{\mu}^{i, 0}\right)=\operatorname{Sh}_{U_{0}^{i}}\left(N_{0}^{i}, v_{\mu}^{i, 0}\right),
\end{aligned}
$$


where the penultimate equality holds by Definition 4.4. Finally,

$$
\begin{aligned}
& \rho_{i}^{\mathrm{Sh}^{\mathrm{L}}}(N, v, \underline{B})=\frac{\mathrm{Sh}_{i}^{\mathrm{L}}(N, v, \underline{B})}{v(N)}=\frac{\mathrm{Sh}_{i}^{\mathrm{L}}(N, v, \underline{B})}{v(N)} \prod_{r=0}^{k-1} \frac{v_{\mu}^{i, r}\left(N_{r}^{i}\right)}{v_{\mu}^{i, r}\left(N_{r}^{i}\right)} \\
& =\frac{\mathrm{Sh}_{i}^{\mathrm{L}}(N, v, \underline{B})}{v(N)} \prod_{r=0}^{k-1} \frac{\mathrm{Sh}_{U_{r+1}^{i}}\left(N_{r+1}^{i}, v_{\mu}^{i, r+1}\right)}{v_{\mu}^{i, r}\left(N_{r}^{i}\right)}=\frac{\mathrm{Sh}_{i}^{\mathrm{L}}(N, v, \underline{B})}{v(N)} \prod_{r=1}^{k-1} \frac{\mathrm{Sh}_{U_{r}^{i}}\left(N_{r}^{i}, v_{\mu}^{i, r}\right)}{v_{\mu}^{i, r}\left(N_{r}^{i}\right)} \frac{\operatorname{Sh}_{U_{k}^{i}}\left(N_{k}^{i}, v_{\mu}^{i, k}\right)}{v_{\mu}^{i, 0}\left(N_{0}^{i}\right)} \\
& =\frac{\mathrm{Sh}_{U_{0}^{i}}\left(N_{0}^{i}, v_{\mu}^{i, 0}\right)}{v_{\mu}^{i, 0}\left(N_{0}^{i}\right)} \prod_{r=1}^{k-1} \frac{\mathrm{Sh}_{U_{r}^{i}}\left(N_{r}^{i}, v_{\mu}^{i, r}\right)}{v_{\mu}^{i, r}\left(N_{r}^{i}\right)} \frac{\operatorname{Sh}_{U_{k}^{i}}\left(N_{k}^{i}, v_{\mu}^{i, k}\right)}{v_{\mu}^{i, k}\left(N_{k}^{i}\right)}=\prod_{r=0}^{k} \rho_{U_{r}^{i}}^{\mathrm{Sh}}\left(N_{r}^{i}, v_{\mu}^{i, r}\right),
\end{aligned}
$$

where the third equality holds by eq. (15), the fifth equality holds by eq. (16) and the fact that $v_{\mu}^{i, k}\left(N_{k}^{i}\right)=v(N)$, and the last equality holds by the definition of $\rho^{\mathrm{Sh}}$.

In Álvarez-Mozos and Tejada (2011) a generalization of the Banzhaf value is introduced and characterized for games with levels structure of cooperation. This Banzhaf value generalizes the Owen-Banzhaf value given in Owen (1981) for games with coalition structure. From van der Laan and van den Brink (2002) it follows that the corresponding share function does not belong to their class of CS-share functions. Therefore, the share function corresponding to the Banzhaf levels value of Álvarez-Mozos and Tejada (2011) does not belong to the class of LS-share functions defined in this section. In particular it does not satisfy the multiplication property, that we introduce in the next section.

\section{Characterization of the class of LS-share functions}

The characterization of a share function consists of finding a minimal set of properties that uniquely determine it. In many situations characterizing a share function is more appealing than just giving an explicit formula or procedure to calculate it. In this section we propose sets of properties that characterize the class of $\mu$-LS-share functions. We consider two types of properties. Properties of the first type apply only to games with the trivial levels structure of cooperation, whereas properties of the second type involve games with arbitrary levels structures of cooperation.

The following properties of the first type are standard in the literature for games without restrictions on the cooperation possibilities and are based on NPP, SYM and $\mu$-ADD properties defined in Section 3.2.

$\mathrm{NPP}^{0}$ A LS-share function $\rho$ satisfies the null player property for trivial levels structures of cooperation if, for every $\left(N, v, \underline{B_{0}}\right) \in \mathcal{G L}$ with $v \neq v_{0}$ and every null player $i \in N$ in $(N, v)$, 


$$
\rho_{i}\left(N, v, \underline{B_{0}}\right)=0
$$

SYM $^{0}$ A LS-share function $\rho$ satisfies symmetry for trivial levels structures of cooperation if, for every $\left(N, v, \underline{B_{0}}\right) \in \mathcal{G} \mathcal{L}$ and every pair $i, j \in N$ of symmetric players in $(N, v)$,

$$
\rho_{i}\left(N, v, \underline{B_{0}}\right)=\rho_{j}\left(N, v, \underline{B_{0}}\right)
$$

$\mu$-ADD ${ }^{0}$ Let $\mu: \mathcal{G} \rightarrow \mathbb{R}$. A LS-share function $\rho$ satisfies $\mu$-additivity for trivial levels structures of cooperation if, for every pair $\left(N, v, \underline{B_{0}}\right),\left(N, w, \underline{B_{0}}\right) \in \mathcal{G L}$,

$$
\mu(N, v+w) \rho\left(N, v+w, \underline{B_{0}}\right)=\mu(N, v) \rho\left(N, v, \underline{B_{0}}\right)+\mu(N, w) \rho\left(N, w, \underline{B_{0}}\right) .
$$

Next, we consider properties of the second type, which apply to arbitrary games with levels structure of cooperation.

$\mu$-MUL Let $\mu: \mathcal{G} \rightarrow \mathbb{R}$. A LS-share function, $\rho$, satisfies $\mu$-multiplication if for every $(N, v, \underline{B}) \in$ $\mathcal{G L}$ and $i \in N$,

$$
\rho_{i}(N, v, \underline{B})=\prod_{r=0}^{k} \rho_{U_{r}^{i}}\left(N_{r}^{i}, v_{\mu}^{i, r}, \underline{B_{0}}\right) .
$$

$\mu$-ADD ${ }^{\mathrm{L}}$ Let $\mu: \mathcal{G} \rightarrow \mathbb{R}$. A LS-share function $\rho$ satisfies $\mu$-additivity for arbitrary levels structures of cooperation if for every pair of games $(N, v, \underline{B}),(N, w, \underline{B}) \in \mathcal{G} \mathcal{L}$, and every player $i \in N$,

$$
\begin{aligned}
& \rho_{i}(N, v+w, \underline{B}) \cdot \prod_{r=0}^{k} \mu\left(N_{r}^{i},(v+w)_{\mu}^{i, r}\right) \\
& =\prod_{r=0}^{k}\left[\mu\left(N_{r}^{i}, v_{\mu}^{i, r}\right) \rho_{U_{r}^{i}}\left(N_{r}^{i}, v_{\mu}^{i, r}, \underline{B_{0}}\right)+\mu\left(N_{r}^{i}, w_{\mu}^{i, r}\right) \rho_{U_{r}^{i}}\left(N_{r}^{i}, w_{\mu}^{i, r}, \underline{B_{0}}\right)\right] .
\end{aligned}
$$

CON A LS-share function $\rho$ satisfies consistency ${ }^{5}$ if, for every $(N, v, \underline{B}) \in \mathcal{G} \mathcal{L}$ and every $U \in B_{r}$ for some $r \in\{1, \cdots, k\}$,

$$
\sum_{i \in U} \rho_{i}(N, v, \underline{B})=\rho_{U}\left(B_{r}, v^{r}, \underline{B^{r}}\right) .
$$

$\mu$-FAIR Let $\mu: \mathcal{G} \rightarrow \mathbb{R}$. A LS-share function $\rho$ satisfies $\mu$-fairness if, for every $(N, v, \underline{B}) \in \mathcal{G} \mathcal{L}$ and every union of the first level, $U \in B_{1}$, there is a scalar $K_{U,\left(B_{1}, v^{1}, B_{1}\right)}^{\mu} \in \mathbb{R}_{+}$such that, for every $i, j \in U$,

\footnotetext{
${ }^{5}$ We would like to mention that the term "consistency" has also been applied in the literature to other properties, for instance the reduced game consistency property. Also observe that the property mimics the requirements in Level Game Property, but it applies to share functions instead of values.
} 


$$
\rho_{i}(N, v, \underline{B})-\rho_{j}(N, v, \underline{B})=K_{U,\left(B_{1}, v^{1}, \underline{B_{1}}\right.}^{\mu}\left[\rho_{i}\left(U, v_{\mu}^{i, 0}, \underline{B_{0}}\right)-\rho_{j}\left(U, v_{\mu}^{j, 0}, \underline{B_{0}}\right)\right] .
$$

Property $\mu$-MUL implies that the share of a player in a game with levels structure of cooperation is obtained by sequentially multiplying her share in the lowest level game with the share of the union she belongs to on the first level in the second level game, and so on and so forth. It generalizes a principle mentioned by Owen (1977) when introducing his Owen value for games with coalition structures and used by van den Brink and van der Laan (2005) to characterize a class of CS-share functions. By Definition 4.6 it is obvious that $\mu$-LS-share functions satisfy this property, but here we state it as an explicit axiom that a solution for games with levels structure of cooperation (in terms of share functions) should satisfy. Similarly, CON is based on a property that is introduced by Owen (1977) as a desirable requirement for a solution for games with coalition structure, and is satisfied by the class of CS-share functions according to van den Brink and van der Laan (2005). It states that the sum of the shares of the players in a union at any level $r$ equals the share of this union in the game between the unions on this level in the game $\left(B_{r}, v^{r}, \underline{B_{r}}\right)$. It is obvious from Proposition 4.7.(iv) that all $\mu$-LS-share functions satisfy this property. By Proposition 4.7.(v), the $\mu$-LS-share functions satisfy $\mu$-ADD ${ }^{\mathrm{L}}$, which again generalizes two properties considered in van den Brink and van der Laan (2005) for games with coalition structure. Finally, $\mu$-FAIR is a new axiom for games with levels structure of cooperation. It has some similarity with fairness or the equal gain/loss principle that can be found in various parts of the game theory literature. However, in this case we do not change the game or structure (which is usual in such fairness axioms), but we compare the difference between the shares of two players who are symmetric in the structure (but not necessarily in the game) with the difference between their shares in the game $v_{\mu}^{i, 0}$ on the zero level, where the constant only depends on $\mu$ and the first level game. We now state and prove the first characterization result.

Theorem 5.1. Let $\mu: \mathcal{G} \rightarrow \mathbb{R}$ be additive, positive, and anonymous. Then, $\rho^{\mu}$ is the unique LSshare function on $\mathcal{G} \mathcal{L}$ that satisfies $\mathrm{NPP}^{0}, \mathrm{SYM}^{0}, \mu$ - $\mathrm{ADD}^{0}$, and $\mu$-MUL. Moreover, the properties are independent.

Proof. It has been shown in Proposition 4.7 that $\rho^{\mu}$ satisfies the four properties (notice that $\mu$-ADD $D^{\mathrm{L}}$ implies $\mu$-ADD ${ }^{0}$. Thus we only need to check that $\rho^{\mu}$ is the unique LS-share function satisfying the properties.

Let $\rho$ be a LS-share function satisfying the properties. For games with trivial levels structures of cooperation $\rho$ is characterized in Proposition 3.3 as the unique share function that 
satisfies $\mathrm{NPP}^{0}, \mathrm{SYM}^{0}$, and $\mu$-ADD ${ }^{0}$. Then, $\mu$-MUL relates the share of any player in any game with levels structure of cooperation to shares of players in games with the trivial levels structure of cooperation. Hence, by $\mu$-MUL and the uniqueness for trivial levels structures, $\rho$ is unique.

Finally, we show that the properties are independent by means of the following examples.

(i) The LS-share function $\rho^{1}$ defined, for every $(N, v, \underline{B}) \in \mathcal{G} \mathcal{L}$, by $\rho^{1}(N, v, \underline{B})=\rho^{\mu}(N, v)$ satisfies $\mathrm{NPP}^{0}, \mathrm{SYM}^{0}$, and $\mu$-ADD ${ }^{0}$ but not $\mu$-MUL.

(ii) The LS-share function $\rho^{2}$ defined, for every $(N, v, \underline{B}) \in \mathcal{G L}$ by $\rho^{2}(N, v, \underline{B})=\prod_{r=1}^{k} \frac{1}{\left|N_{r}^{i}\right|}$, satisfies $\mathrm{SYM}^{0}, \mu-\mathrm{ADD}^{0}$, and $\mu$-MUL but not $\mathrm{NPP}^{0}$.

(iii) Let $a$ and $b$ be two different and fixed players and $N=\{a, b\}$. We know that there are $\lambda_{1}, \lambda_{2}>0$ such that $\mu(N, v)=\lambda_{1} v(a)+\lambda_{1} v(b)+\lambda_{2} v(N)$. Then, define the LS-share function $\rho^{3}$, for every $(N, v, \underline{B}) \in \mathcal{G} \mathcal{L}$, as

- If $N=\{a, b\}, \underline{B}=\underline{B_{0}}$, and $(N, v) \in \mathcal{G}_{+}$,

$$
\left\{\begin{array}{l}
\rho_{a}^{3}(N, v, \underline{B})=\frac{1}{\mu(N, v)}\left[\lambda_{1} v(a)+\lambda_{2}(v(N)-v(b))\right] \\
\rho_{b}^{3}(N, v, \underline{B})=\frac{1}{\mu(N, v)}\left(\lambda_{1}+\lambda_{2}\right) v(b)
\end{array}\right.
$$

- If $\underline{B}=\underline{B_{0}}$ and $N \neq\{a, b\}, \rho^{3}(N, v, \underline{B})=\rho^{\mu}(N, v)$.

- If $\underline{B} \neq \underline{B_{0}}$ for every $i \in N, \rho_{i}^{3}(N, v, \underline{B})=\prod_{r=0}^{k} \rho_{U_{r}^{i}}^{3}\left(N_{r}^{i}, v_{\mu}^{i, r}, \underline{B_{0}}\right)$.

Then, $\rho^{3}$ satisfies $\mathrm{NPP}^{0}, \mu$-ADD ${ }^{0}$, and $\mu$-MUL but not $\mathrm{SYM}^{0}$.

(iv) Let $\mu^{0}: \mathcal{G} \rightarrow \mathbb{R}$ be additive, positive, anonymous, and different from $\mu$. The LS-share function $\rho^{4}$ defined, for every $(N, v, \underline{B}) \in \mathcal{G} \mathcal{L}$ and $i \in N$, by

$$
\rho_{i}^{4}(N, v, \underline{B})=\prod_{r=0}^{k} \rho_{U_{r}^{i}}^{\mu^{0}}\left(N_{r}^{i}, v_{\mu}^{i, r}\right),
$$

satisfies $\mathrm{SYM}^{0}, \mathrm{NPP}^{0}$, and $\mu$-MUL but not $\mu-\mathrm{ADD}^{0}$.

Theorem 5.1 upgrades Theorems 4.5 and 4.8 in van den Brink and van der Laan (2005) in two ways. First, it extends the two characterization results from Coalition Structure (being a levels structure with $k=1$ ) share functions to LS-share functions. Second, it replaces for both characterizations three of the axioms by weaker versions by requiring them only for trivial levels structures. 
Obviously, $\mu-\mathrm{ADD}^{\mathrm{L}}$ implies $\mu-\mathrm{ADD}^{0}$, which can be seen by taking $\underline{B}=\underline{B_{0}}$. Moreover, $\mu$-ADD ${ }^{\mathrm{L}}$ implies $\mu$-MUL, which can be seen by taking $w=v_{0}$. From Proposition 4.7.(v) it follows that every LS-share function $\rho^{\mu}$ satisfies $\mu$-ADD ${ }^{\mathrm{L}}$. Therefore, Theorem 5.1 yields the following corollary.

Corollary 5.2. Let $\mu: \mathcal{G} \rightarrow \mathbb{R}$ be additive, positive, and anonymous. Then, the function $\rho^{\mu}$ is the unique LS-share function that satisfies $\mathrm{NPP}^{0}, \mathrm{SYM}^{0}$, and $\mu$ - $\mathrm{ADD}^{\mathrm{L}}$. Moreover, the properties are independent.

Using the LS-share functions considered in the proof of Theorem 5.1 it can be shown that the properties considered in Corollary 5.2 are also independent. Indeed, the share function $\rho^{1}$ satisfies $\mathrm{NPP}^{0}$ and $\mathrm{SYM}^{0}$ but not $\mu$-ADD ${ }^{\mathrm{L}}$, the share function $\rho^{2}$ satisfies $\mathrm{SYM}^{0}$ and $\mu$-ADD ${ }^{\mathrm{L}}$ but not $\mathrm{NPP}^{0}$, and the share function $\rho^{3}$ satisfies $\mathrm{NPP}^{0}$ and $\mu$-ADD ${ }^{\mathrm{L}}$ but not $\mathrm{SYM}^{0}$. Corollary 5.2 upgrades Theorem 5.3 in van den Brink and van der Laan (2005) in two ways by first extending the characterization result of the LS-share function from one-level structures to an arbitrary number of levels structures of cooperation, and second by replacing two of the axioms by weaker versions that apply only to trivial structures. Moreover, it shows that the consistency property used in van den Brink and van der Laan (2005) can be dropped without changing the result.

Finally, we give a characterization of $\rho^{\mu}$ by using the CON and $\mu$-FAIR axioms.

Theorem 5.3. Let $\mu: \mathcal{G} \rightarrow \mathbb{R}$ be additive, positive and anonymous. Then, $\rho^{\mu}$ is the unique LSshare function that satisfies $\mathrm{NPP}^{0}, \mathrm{SYM}^{0}, \mu-\mathrm{ADD}^{0}$, $\mathrm{CON}$, and $\mu$-FAIR. Moreover, the properties are independent.

Proof. It has been shown in Proposition 4.7 that $\rho^{\mu}$ satisfies the first four properties. By definition of $\rho^{\mu}$ we have that

$$
\rho_{i}(N, v, \underline{B})=\prod_{r=0}^{k} \rho_{U_{r}^{i}}^{\mu}\left(N_{r}^{i}, v_{\mu}^{i, r}, \underline{B_{0}}\right)=\rho_{U_{0}^{i}}^{\mu}\left(N_{0}^{i}, v_{\mu}^{i, 0}, \underline{B_{0}}\right) \cdot \prod_{r=1}^{k} \rho_{U_{r}^{i}}^{\mu}\left(N_{r}^{i}, v_{\mu}^{i, r}, \underline{B_{0}}\right)
$$

Now, for some $U \in B_{1}$, let $i, j$ be two players in $U$. Then $U_{0}^{i}=\{i\}, U_{0}^{j}=\{j\}, N_{0}^{i}=N_{0}^{j}=U$, and $U_{r}^{i}=U_{r}^{j}, N_{r}^{i}=N_{r}^{j}$ and $v_{\mu}^{i, r}=v_{\mu}^{j, r}$ for all $r \in\{1, \ldots, k\}$. From substituting this in the equation above for players $i$ and $j$ respectively, and the fact that $\prod_{r=1}^{k} \rho_{U_{r}^{i}}^{\mu}\left(N_{r}^{i}, v_{\mu}^{i, r}, \underline{B_{0}}\right)=\rho_{U}^{\mu}\left(B_{1}, v^{1}, \underline{B_{1}}\right)$, it follows that $\rho^{\mu}$ satisfies $\mu$-FAIR.

In order to prove uniqueness, suppose that $\rho^{1}$ and $\rho^{2}$ are two LS-share functions satisfying the properties. We show that for every $(N, v, \underline{B}) \in \mathcal{G} \mathcal{L}, \rho^{1}(N, v, \underline{B})=\rho^{2}(N, v, \underline{B})$ by induction on the number of levels $k$. If $k=0$, then $\underline{B}=\underline{B_{0}}$. In this case, by $\mathrm{NPP}^{0}, \mathrm{SYM}^{0}$, and $\mu$ $\mathrm{ADD}^{0}$, from Proposition 3.3 we have that $\rho^{1}(N, v, \underline{B})=\rho^{2}(N, v, \underline{B})=\rho^{\mu}(N, v)$. Next, assume 
that $\rho^{1}\left(N^{\prime}, v^{\prime}, \underline{B^{\prime}}\right)=\rho^{2}\left(N^{\prime}, v^{\prime}, \underline{B^{\prime}}\right)$ for every $\left(N^{\prime}, v^{\prime}, \underline{B^{\prime}}\right) \in \mathcal{G} \mathcal{L}$ with at most $k-1$ levels of cooperation, and let $(N, v, \underline{B}) \in \mathcal{G} \mathcal{L}$ with $k$ levels of cooperation. Let also $U \in B_{1}$. On the one hand, if $|U|=1$ then for the only player $i \in U$ we have that $\rho_{i}(N, v, \underline{B})=\rho_{U}\left(B_{1}, v^{1}, \underline{B_{1}}\right)$ has been determined uniquely by the induction hypotheses. On the other hand, if $|U| \geq 2$, let $i$ be one of the players of $U$. Then, for every other player $j$ in $U$, by $\mu$-FAIR there is a scalar $K_{U,\left(B_{1}, v^{1}, \underline{B_{1}}\right)}^{\mu} \in \mathbb{R}_{+}$such that,

$$
\begin{aligned}
& \rho_{i}^{1}(N, v, \underline{B})-\rho_{j}^{1}(N, v, \underline{B})=K_{U,\left(B_{1}, v^{1}, \underline{B_{1}}\right)}^{\mu}\left[\rho_{i}^{1}\left(U, v_{\mu}^{i, 0}, \underline{B_{0}}\right)-\rho_{j}^{1}\left(U, v_{\mu}^{j, 0}, \underline{B_{0}}\right)\right] \\
& =K_{U,\left(B_{1}, v^{1}, \underline{B_{1}}\right)}^{\mu}\left[\rho_{i}^{2}\left(U, v_{\mu}^{i, 0}, \underline{B_{0}}\right)-\rho_{j}^{2}\left(U, v_{\mu}^{j, 0}, \underline{B_{0}}\right)\right]=\rho_{i}^{2}(N, v, \underline{B})-\rho_{j}^{2}(N, v, \underline{B}),
\end{aligned}
$$

where the second equality is a consequence of the uniqueness for games with trivial levels structure of cooperation. Next, for the given player $i$, adding up eq. (17) for every $j \in U$, including $i$, we obtain

$$
\left|U_{1}^{i}\right| \rho_{i}^{1}(N, v, \underline{B})-\sum_{j \in U_{1}^{i}} \rho_{j}^{1}(N, v, \underline{B})=\left|U_{1}^{i}\right| \rho_{i}^{2}(N, v, \underline{B})-\sum_{j \in U_{1}^{i}} \rho_{j}^{2}(N, v, \underline{B}) .
$$

By CON it follows that

$$
\sum_{j \in U_{1}^{i}} \rho^{1}(N, v, \underline{B})=\rho_{U_{1}^{i}}^{1}\left(B_{1}, v^{1}, \underline{B_{1}}\right)=\rho_{U_{1}^{i}}^{2}\left(B_{1}, v^{1}, \underline{B_{1}}\right)=\sum_{j \in U_{1}^{i}} \rho^{2}(N, v, \underline{B}),
$$

where the second equality is due to the induction hypothesis, since $\left|\underline{B_{1}}\right|=k-1$. This last equation together with eq. (18) concludes the proof that $\rho$ is uniquely determined.

Finally, we show the independence of the properties. Indeed,

(i) The LS-share function, $\rho^{2}$, defined as in the proof of Theorem 5.1 satisfies $\mathrm{SYM}^{0}, \mu$-ADD ${ }^{0}$, CON, and $\mu$-FAIR but not NPP ${ }^{0}$.

(ii) The LS-share function, $\rho^{3}$, defined as in the proof of Theorem 5.1 satisfies $\mathrm{NPP}^{0}, \mu$-ADD ${ }^{0}$, CON, and $\mu$-FAIR but not $\mathrm{SYM}^{0}$.

(iii) The LS-share function, $\rho^{4}$, defined as in the proof of Theorem 5.1 satisfies $\mathrm{NPP}^{0}, \mathrm{SYM}^{0}$, CON, and $\mu$-FAIR but not $\mu$-ADD ${ }^{0}$.

(iv) Let $\rho^{5}$ be the LS-share function defined for every $(N, v, \underline{B}) \in \mathcal{G} \mathcal{L}$ as follows:

$$
\text { - If } \underline{B}=\underline{B_{0}} \text {, }
$$

$$
\rho^{5}(N, v, \underline{B})=\rho^{\mu}(N, v) .
$$


- Otherwise, for every $i \in N$

$$
\rho_{i}^{5}(N, v, \underline{B})=\rho_{U_{1}^{i}}^{\mu}\left(B_{1}, v^{1}, \underline{B_{1}}\right) \cdot \frac{1}{\left|U_{1}^{i}\right|} .
$$

Then $\rho^{5}$ satisfies $\mathrm{NPP}^{0}, \mathrm{SYM}^{0}, \mu$-ADD ${ }^{0}$, and $\mu$-FAIR but not $\mathrm{CON}$.

(v) Let $\rho^{6}$ be the LS-share function defined for every $(N, v, \underline{B}) \in \mathcal{G} \mathcal{L}$ as follows:

- If $\underline{B}=\underline{B_{0}}$,

$$
\rho^{6}(N, v, \underline{B})=\rho^{\mu}(N, v) .
$$

- If $N$ is a set of indivisible players, that is for every $i \in N$ there are no players $i_{1}, \ldots, i_{l} \in N^{\prime}$ such that $i=\left\{i_{1}, \ldots, i_{l}\right\}$. For every $U \in B_{1}$ let $i_{U} \in U$ be a randomly selected particular agent, then

$$
\left\{\begin{array}{l}
\rho_{i_{U}}^{6}(N, v, \underline{B})=\underline{\rho_{U}^{\mu}}\left(B_{1}, v^{1}, \underline{B_{1}}\right) \\
\rho_{i}^{6}(N, v, \underline{B})=0 \quad \text { for every } i \in U \backslash i_{U}
\end{array}\right.
$$

- Otherwise,

$$
\rho_{i}^{6}(N, v, \underline{B})=\underline{\rho_{i}^{\mu}}(N, v, \underline{B}) .
$$

Then $\rho^{6}$ satisfies $\mathrm{NPP}^{0}, \mathrm{SYM}^{0}, \mu$-ADD ${ }^{0}$, and $\mathrm{CON}$ but not $\mu$-FAIR.

It is worth noting that when we consider $\mu^{\text {Sh }}$, Theorems 5.1 and 5.3 and Corollary 5.2, together with Proposition 4.10, provide us with three different characterization results of the Shapley levels share function $\rho^{\mathrm{Sh}^{\mathrm{L}}}$.

\section{Appendix}

Proposition 6.1. A mapping $\mu: \mathcal{G} \rightarrow \mathbb{R}$ is symmetric if and only if it is anonymous.

Proof. On the one hand, let $\mu: \mathcal{G} \rightarrow \mathbb{R}$ be anonymous. Let also $i, j \in N$ be two symmetric players in $(N, v) \in \mathcal{G}$ and $S \subseteq N \backslash\{i, j\}$. Consider the permutation $\pi \in \Pi(N)$ that leaves any player in $N \backslash\{i, j\}$ invariant and exchanges player $i$ with player $j$, i.e., $\pi(i)=j, \pi(j)=$ $i$, and $\pi(k)=k$ for all $k \in N \backslash\{i, j\}$. This type of permutations are known as transpositions. We denote the set of all transpositions over $N$ by $\Pi^{*}(N)$. Then, since $\mu$ is anonymous,

$$
\mu\left(S \cup i, v_{\mid S \cup i}\right)=\mu\left(\pi(S \cup i),\left(\pi^{-1} v\right)_{\mid \pi(S \cup i)}\right) .
$$


By definition, for every $T \subseteq \pi(S \cup i)=S \cup j$,

$$
\left(\pi^{-1} v\right)(T)=v\left(\pi^{-1}(T)\right)= \begin{cases}v((T \backslash j) \cup i) & \text { if } j \in T, \\ v(T) & \text { if } j \notin T,\end{cases}
$$

which, means that $\left(\pi(S \cup i),\left(\pi^{-1} v\right)_{\mid \pi(S \cup i)}\right)=\left(S \cup j, v_{\mid S \cup j}\right)$ because $i$ and $j$ are symmetric players in $(N, v)$. Therefore, by eq. (19), $\mu$ is symmetric.

On the other hand, let $\mu: \mathcal{G} \rightarrow \mathbb{R}$ be symmetric. Let also $\pi \in \Pi(N)$. Since it is otherwise straightforward, we assume that $|N| \geq 2$ and $\pi$ is not the identity permutation, that is, the permutation that leaves every player invariant. It is well known that any such permutation can be written as a finite composition of transpositions, i.e., there are $\pi_{1}, \ldots, \pi_{r} \in \Pi^{*}(N)$ such that

$$
\pi=\pi_{1} \circ \cdots \circ \pi_{r} .
$$

We claim that for every $(N, v) \in \mathcal{G}, \pi \in \Pi^{*}(N)$, and $\emptyset \neq S \subseteq N$,

$$
\mu\left(S, v_{\mid S}\right)=\mu\left(\pi(S),\left(\pi^{-1} v\right)_{\mid \pi(S)}\right)
$$

Observe that if eq. (20) holds then $\mu$ is anonymous. Indeed, let $(N, v) \in \mathcal{G}, \pi \in \Pi(N)$, and $\emptyset \neq S \subseteq N$. Let also $\pi=\pi_{1} \circ \cdots \circ \pi_{r}$ be a decomposition of $\pi$ in transpositions. Then,

$$
\begin{aligned}
\mu\left(S, v_{\mid S}\right) & =\mu\left(\pi_{r}(S),\left(\pi_{r}^{-1} v\right)_{\mid \pi_{r}(S)}\right)=\mu\left(\pi_{r-1}\left(\pi_{r}(S)\right),\left(\pi_{r-1}^{-1}\left(\pi_{r}^{-1} v\right)\right)_{\mid \pi_{r-1}\left(\pi_{r}(S)\right)}\right) \\
& =\cdots=\mu\left(\pi_{1}\left(\cdots\left(\pi_{r}(S)\right) \cdots\right),\left(\pi_{1}^{-1}\left(\cdots\left(\pi_{r}^{-1} v\right) \cdots\right)\right)_{\mid \pi_{1}\left(\cdots\left(\pi_{r}(S)\right) \cdots\right)}\right) \\
& =\mu\left(\left(\pi_{1} \circ \cdots \circ \pi_{r}\right)(S),\left(\left(\pi_{1} \circ \cdots \circ \pi_{r}\right)^{-1} v\right)_{\mid \pi_{1} \circ \cdots \circ \pi_{r}(S)}\right)=\mu\left(\pi(S),\left(\pi^{-1} v\right)_{\mid \pi(S)}\right) .
\end{aligned}
$$

Hence, it only remains to prove eq. (20). Let $\pi \in \Pi^{*}(N)$ be a transposition that exchanges $i$ with $j$ for some $i, j \in N$ and let $\emptyset \neq S \subseteq N$. We distinguish three cases.

Case 1: $S \cap\{i, j\}=\emptyset$.

Since $\pi_{\mid S}$ is the identity permutation, eq. (20) is trivially satisfied.

Case 2: $\emptyset \neq S \cap\{i, j\} \subsetneq\{i, j\}$.

Observe that it is equivalent to say that $S \backslash \pi(S) \neq \emptyset$ and $\pi(S) \backslash S \neq \emptyset$. We assume without loss of generality that $S \cap\{i, j\}=\{i\}$. Then consider the game $\left(N^{\prime}, v^{\prime}\right)$, defined by $N^{\prime}=S \cup j$ and for every $T \subseteq N^{\prime}$, by

$$
v^{\prime}(T)= \begin{cases}v((T \backslash j) \cup i) & \text { if } j \in T, i \notin T, \\ v(T) & \text { if } j \notin T .\end{cases}
$$


By construction, $i$ and $j$ are symmetric players in $\left(N^{\prime}, v^{\prime}\right)$. Let $S^{\prime}=S \backslash i$. Then, by symmetry,

$$
\mu\left(S, v_{\mid S}\right)=\mu\left(S^{\prime} \cup i, v_{\mid S^{\prime} \cup i}^{\prime}\right)=\mu\left(S^{\prime} \cup j, v_{\mid S^{\prime} \cup j}^{\prime}\right)=\mu\left(\pi(S),\left(\pi^{-1} v\right)_{\mid \pi(S)}\right) .
$$

Case 3: $\{i, j\} \subseteq S$.

Observe that in this case we have $\pi(S)=S$. Let $k \notin N$ be an extra (fictitious) player. Then, define the game $\left(N^{\prime}, v^{\prime}\right)$ where $N^{\prime}=N \cup k$ and $v^{\prime}(T)=v(T \backslash k)$ for all $T \subseteq N^{\prime}$. Let also $\pi_{i, k} \in \Pi^{*}\left(N^{\prime}\right)$ be the transposition that exchanges $i$ with $k$ and leaves the remaining players invariant. Similarly, let $\pi_{j, i}, \pi_{j, k} \in \Pi^{*}\left(N^{\prime}\right)$ be the transpositions that exchange $j$ with $i$ and $j$ with $k$, respectively. It is an easy exercise to check that $\pi=\pi_{j, k} \circ \pi_{j, i} \circ \pi_{i, k}$. Let $T=S \backslash\{i, j\}$ and define

$$
\begin{aligned}
& S^{0}=T \cup\{i, j\}=S, \\
& S^{1}=\pi_{i, k}\left(S^{0}\right)=\pi_{i, k}(T \cup\{i, j\})=T \cup\{k, j\}, \\
& S^{2}=\pi_{j, i}\left(S^{1}\right)=\left(\pi_{j, i} \circ \pi_{i, k}\right)(T \cup\{i, j\})=T \cup\{k, i\}, \\
& S^{3}=\pi_{j, k}\left(S^{2}\right)=\left(\pi_{j, k} \circ \pi_{j, i} \circ \pi_{i, k}\right)(T \cup\{i, j\})=T \cup\{i, j\}=S .
\end{aligned}
$$

Observe that $S^{0} \backslash \pi_{i, k}\left(S^{0}\right) \neq \emptyset$ and $\pi_{i, k}\left(S^{0}\right) \backslash S^{0} \neq \emptyset$. Hence, by Case 2,

$$
\mu\left(S^{0}, v_{\mid S^{0}}\right)=\mu\left(\pi_{i, k}\left(S^{0}\right),\left(\pi_{i, k}^{-1} v\right)_{\mid \pi_{i, k}\left(S^{0}\right)}\right) .
$$

Analogously, since $S^{1} \cap \pi_{j, i}\left(S^{1}\right) \backslash \emptyset$ and $\pi_{j, i}\left(S^{1}\right) \backslash S^{1} \neq \emptyset$, we have

$$
\mu\left(S^{1},\left(\pi_{i, k}^{-1} v\right)_{\mid S^{1}}\right)=\mu\left(\pi_{j, i}\left(S^{1}\right),\left(\pi_{j, i}^{-1}\left(\pi_{i, k}^{-1} v\right)\right)_{\mid \pi_{j, i}\left(S^{1}\right)}\right),
$$

whereas, since $S^{2} \cap \pi_{k, j}\left(S^{2}\right) \backslash \emptyset$ and $\pi_{k, j}\left(S^{2}\right) \backslash S^{2} \neq \emptyset$, we have

$$
\begin{aligned}
\mu\left(S^{2},\left(\pi_{j, i}^{-1}\left(\pi_{i, k}^{-1} v\right)\right)_{\mid S^{2}}\right) & =\mu\left(\pi_{j, k}\left(S^{2}\right),\left(\pi_{j, k}^{-1}\left(\pi_{j, i}^{-1}\left(\pi_{i, k}^{-1} v\right)\right)\right)_{\mid \pi_{j, k}\left(S^{2}\right)}\right. \\
& =\mu\left(S^{3},\left(\left(\pi_{j, k} \circ \pi_{j, i} \circ \pi_{i, k}\right)^{-1} v\right)_{\mid S^{3}} .\right.
\end{aligned}
$$

Finally, the claim in eq. (20) follows from eq. (21), eq. (22), and eq. (23).

\section{Acknowledgements}

This research received financial support from the Spanish Ministry of Economy and Competitiveness through projects ECO2008-03484-C02-02/ECON, ECO2008-02344/ECON, and MTM201127731-C03-02 as well as from Xunta de Galicia (Project INCITE09-207-064PR) and Generalitat de Catalunya (Grant 2009SGR0960). Support from the FPU program of the Spanish Ministry 
of Education is also acknowledged. We would also like to thank two referees for their comments and suggestions, which helped improve the presentation of the paper. All remaining errors are only our responsibility.

\section{References}

Aczél, J. and J. Dhombres (1989). Functional equations in several variables. Cambridge University Press.

Albizuri, M.J. and J. Aurrekoetxea (2006). Coalition configurations and the Banzhaf index. Social Choice and Welfare 26, 571-596.

Albizuri, M.J., J. Aurrekoetxea and J.M. Zarzuelo (2006). Configuration values: Extensions of the coalitional Owen value. Games and Economic Behavior 57, 1-17.

Álvarez-Mozos, M. and O. Tejada (2011). Parallel characterizations of a generalized Shapley value and a generalized Banzhaf value for cooperative games with levels structure of cooperation. Decision Support Systems 52, 21-27.

Andjiga, N. and S. Courtin (2010). Coalition configurations and share functions. Technical report. CREM.

Aumann, R. J. and J. Drèze (1974). Cooperative games with coalition structures. International Journal of Game Theory 3, 217-237.

Banzhaf, J. F. (1965). Weighted voting does not work: A mathematical analysis. Rutgers Law Review 19, 317-343.

Brink, R. van den and G. van der Laan (1998). Axiomatizations of the normalized Banzhaf value and the Shapley value. Social Choice and Welfare 15, 567-582.

Brink, R. van den and G. van der Laan (1999). The normalized Banzhaf value and the Banzhaf share function. International Journal of Mathematics, Game Theory, and Algebra 9, 65-84.

Brink, R. van den and G. van der Laan (2005). A class of consistent share functions for games in coalition structure. Games and Economic Behavior 51, 193-212.

Courtin, S. (2011). Power in the European Union: an evaluation according to a priori relations between states. Economics Bulletin 31, 534-545. 
Dubey, P. and L. S. Shapley (1979). Mathematical properties of the Banzhaf power index. Mathematics of Operations Research 4, 99-131.

Laan, G. van der and R. van den Brink (1998). Axiomatizations of a class of share functions fon n-person games. Theory and Decision 44, 117-148.

Laan, G. van der and R. van den Brink (2002). A Banzhaf share function for cooperative games in coalition structure. Theory and Decision 53, 61-86.

Owen, G. (1975). Multilinear Extensions and the Banzhaf value. Naval Research Logistics Quarterly 22, 741-750.

Owen, G. (1977). Values of games with a priori unions. In: Mathematical Economics and Game Theory (R. Henn and O. Moeschlin, Eds.). pp. 76-88. Springer-Verlag.

Owen, G. (1981). Modification of the Banzhaf-Coleman index for games with a priori unions. In: Power, voting and voting power (M.J. Holler, Ed.). pp. 232-238. Physica-Verlag.

Pekec, A. (2001). Meaningful and meaningless solutions for cooperative $n$-person games. European Journal of Operations Research 133, 608-623.

Shapley, L. S. (1953). A value for n-person games. In: Contributions to the Theory of Games II (A. W. Tucker, Ed.). pp. 307-317. Princeton University Press.

Winter, E. (1989). A value for Cooperative Games with Levels Structure of Cooperation. International Journal of Game Theory 18, 227-240. 